Annals of Solid and Structural Mechanics, 1:29—40, 2010.

\title{
Stiffness Reduction and Fracture Evolution of Oblique Matrix Cracks in Composite Laminates
}

\author{
Daniel H. Cortes ${ }^{1}$ and Ever J. Barbero ${ }^{2}$ \\ Mechanical and Aerospace Engineering, West Virginia University, Morgantown, WV \\ 26506.
}

\begin{abstract}
A constitutive model to predict the onset and evolution of matrix cracking and the subsequent stiffness reduction is derived analytically. The formulation is valid for symmetric laminates with otherwise arbitrary stacking sequence and matrix cracks in one or two directions. The proposed model calculates the reduction of the mechanical properties of the damaged laminate as function of crack densities. The onset and evolution of matrix cracks are predicted by the model in terms of undamaged lamina properties and the critical strain energy release rates for modes I and II ( $\mathrm{G}_{\mathrm{IC}}$ and $\left.\mathrm{G}_{\mathrm{IIC}}\right)$. Therefore, there is not need for postulating damage evolution functions and no need for empirically adjusting the associated material parameters. The model formulation was specialized for the particular case of unidirectional loading. Comparison with experimental data showed an excellent prediction of crack initiation and evolution for a variety of laminate stacking sequences. The combination of constitutive and damage evolution equations formed an integrated, mechanistic damage model with no adjustable parameters.
\end{abstract}

key words: Composites, Matrix Cracks, Stiffness Reduction, Damage Evolution.

\section{Introduction}

Laminate composite materials are prone to variety of failure modes. However, matrix cracking parallel to the fiber direction on off-axis plies is usually the first damage mode observed for in-plane loading. Although matrix cracking is not a catastrophic failure mode, its presence triggers the initiation of other damage modes such as delamination, fiber-matrix debonding, and fiber breakage that usually lead to fracture. Furthermore, matrix cracks drastically increase the permeability of the material, thus allowing access to liquid and gas contaminants that may degrade the fibers and the fiber-matrix interface. Also, it facilitates leakage of liquid and gas that would otherwise be contained in tanks, pressure vessels, and other similar structures. In addition, matrix cracks lead to stiffness reduction and stress redistribution to adjacent laminae. Therefore, the understanding and prediction of matrix cracking has held considerable attention in the literature.

\footnotetext{
${ }^{1}$ Graduate Research Assistant

${ }^{2}$ Professor and Chairman, corresponding author. The original publication is available at www.springerlink.com : http://dx.doi.org/10.1007/s12356-009-0001-5
} 
Annals of Solid and Structural Mechanics, 1:29—40, 2010.

Matrix cranking is usually observed when a tensile stress perpendicular to the fibers is present. Usually, unidirectional loading of cross-ply laminates produces matrix cracking of the $90^{\circ}$ ply when the load is applied in $0^{\circ}$ direction. However this not the only case. Varna et al. (1999) found in experiments for balanced $\left[0 / \pm \theta_{4} / 0_{1 / 2}\right] \mathrm{s}$ laminates that matrix cracks appear for angles as low as $\theta=40$. Yokozeki et al. (2005a, 2005b) observed matrix cracking for ply angles as low as $30^{\circ}$ for unbalanced [0/ $\left.\theta / 90\right]$ s laminates. In addition to this, they showed matrix cracking initiation due to matrix cracks in adjacent plies. Other experimental observations can be found in several reviews (Nairn 2000; Nairn and $\mathrm{Hu} 2004)$.

Continuum Damage Mechanics (CDM) is a common methodology for the prediction of the stiffness reduction in the presence of one or several damage modes. In CDM, damage is represented by internal state variables. Evolution equations for these variables are postulated in terms of additional parameters, which must be adjusted for each material and each configuration using experiments. A review on this methodology can found on Talreja (1994) and Barbero (2007), with more recent publications included in (Miami, et al. 2007a; Li, et al. 1998; Barbero, et al. 2005; Barbero and DeVivo 2001; Barbero and Lonetti 2002, 2001; Lonetti, et al. 2003; Miami, et al. 2007b; Schuecker and Pettermann 2006). A disadvantage of CDM is the difficulty in obtaining the model parameters from experimental data. This issue have been partially solved by combining CDM with other techniques such as micromechanics (Lundmark and Varna 2005; Singh and Talreja 2008; Varna et al. 2001b; Varna et al. 2001a). Other methods include the use of fracture mechanics (Joffe et al. 2001) and in-situ damage effective functions (Kashtalyan and Soutis 2002). Another approach is the analytical solution of the stress and strains in a representative volume cell (Nuismer and Tan 1988; Yokozeki and Aoki 2005), this method is particularly interesting because the material properties of the damaged laminate depend exclusively on the crack density and no additional parameters or functions are needed. However, the solutions presented in (Nuismer and Tan 1988; Yokozeki and Aoki 2005) are limited to very particular cases. In (Nuismer and Tan 1988) the Laminate Stacking Sequence (LSS) is restricted to [0/90n $]$ s where only the 90-lamina can damage. In (Yokozeki and Aoki 2005) the LSS is restricted to $\left[\theta_{n} / \varphi_{m}\right] s$ with cracks on both directions.

Once the mechanical behavior of damaged laminates is modeled by a constitutive equation, a damage evolution equation has to be defined. Several failure criteria have been proposed for the cracking initiation (Camanho, et al. 2006; Davila, et al. 2005; Kashtalyan and Soutis 2007) based on strength models and energy balance methods. Other studies were focused on the matrix cracking evolution as a function of the applied load (Katerelos, et al. 2007; Li, et al. 1998; Tan and Nuismer 1989; Zhang, et al. 1992). Failure criteria are usually based on stress, strain, or strain energy present in the laminate during loading. Therefore, the prediction of damage evolution depends on how accurately the elastic properties of the cracked plate can be predicted.

In this study, an analytical model is proposed to predict both stiffness reduction and matrix cracking evolution for symmetric laminates with arbitrary stacking sequence and cracks in one or two directions. The proposed model calculates the reduction of the 
Annals of Solid and Structural Mechanics, 1:29—40, 2010.

mechanical properties due to matrix cracking as a function of crack densities only. Although previous studies have also proposed similar constitutive relations for the stiffness reduction, in this study the procedure has been generalized to consider arbitrary stacking sequences. Additionally, a damage evolution formulation, which allows prediction of the crack densities as a function of the applied load is derived. An important characteristic of the proposed model is that the crack evolution is determined based on the critical values of the strain energy release rate for modes I and II ( $\mathrm{G}_{\mathrm{IC}}$ and $\mathrm{G}_{\mathrm{IIC}}$ ). Therefore, there is not need for postulating damage evolution functions and no need for empirically adjusting the associated material parameters. Consequently, a complete damage model is obtained by combining the constitutive relations and the damage evolution formulation.

\section{Stiffness Reduction}

In this section the overall stiffness reduction of a laminated composited is studied analytically. The analysis presented here is valid for a symmetric composite with a general LSS and matrix cracks parallel to the fibers, oriented at one or two off-axis angles. A Repetitive Unit Cell (RUC) is chosen as the volume enclosed by the laminate mid surface, laminate top surface and by the parallelogram formed by the crossing of two successive cracks oriented at angles $\varphi$ and $\theta$ (Fig. 1). The lengths $2 l_{1}$ and $2 l_{2}$ of the sides of parallelograms depend on the crack densities $\lambda_{1}$ and $\lambda_{2}$ for cracks at angles $\varphi$ and $\theta$, respectively, as follows

$$
\begin{aligned}
& \lambda_{1}=1 /\left|2 l_{1} \sin (\theta-\varphi)\right| \\
& \lambda_{2}=1 /\left|2 l_{2} \sin (\theta-\varphi)\right|
\end{aligned}
$$

Orthogonal and oblique global coordinate systems, respectively denoted by $X-Y-Z$ and $x$ $y-z$ are used for the present analysis. The $x$ and $y$ axes for the oblique coordinate system are aligned with the two set of matrix cracks (Fig. 1). When only one crack orientation is considered, one in-plane axis is aligned with the cracks and the other in-plane axis can be arbitrarily oriented. Two coordinate transformations (covariant and contravariant) can be defined between the orthogonal and oblique coordinate systems for second-order tensors (Fung 1965)

$$
\begin{gathered}
\mathbf{A}=\mathbf{T} \tilde{\mathbf{A}} \mathbf{T}^{T} \quad \text { (covariant) } \\
\mathbf{A}=\mathbf{T}^{-T} \tilde{\mathbf{A}} \mathbf{T}^{-1} \text { (contravariant) } \\
\mathbf{T}=\left[\begin{array}{ccc}
\cos \theta & \sin \theta & 0 \\
\cos \varphi & \sin \varphi & 0 \\
0 & 0 & 1
\end{array}\right] .
\end{gathered}
$$

In order to obtain physically valid expressions, displacements and strains transformations were chosen to be covariant, and forces and stresses transformations to be contravariant. Transformation of the mechanical properties to the oblique coordinate system can be done using Voigt notation for strains and stresses (see Appendix A). Hereinafter, the procedure to obtain the stiffness reduction due to matrix cracks is presented in the oblique coordinate system. The following assumptions were adopted. Due to the stress-free conditions on the top and bottom surfaces of a thin laminate we have 
Annals of Solid and Structural Mechanics, 1:29—40, 2010.

$$
\sigma_{z}^{(i)}=0
$$

where $(i)$ denotes the $i^{\text {th }}$ lamina. Due to symmetry of the LSS we have

$$
\frac{\partial w^{(i)}}{\partial x}=\frac{\partial w^{(i)}}{\partial y}=0
$$

where $w^{(i)}$ is displacement in the $z$ direction of the $i^{\text {th }}$ lamina. Finally, a linear variation of shear stress in the $z$ direction is assumed

$$
\begin{aligned}
& \tau_{x z}^{(i)}=\tau_{x z}^{i-1, i}+\left(\tau_{x z}^{i, i+1}-\tau_{x z}^{i-1, i}\right) \frac{\left(z-z^{i-1, i}\right)}{h^{(i)}} \\
& \tau_{y z}^{(i)}=\tau_{y z}^{i-1, i}+\left(\tau_{y z}^{i, i+1}-\tau_{y z}^{i-1, i}\right) \frac{\left(z-z^{i-1, i}\right)}{h^{(i)}}
\end{aligned}
$$

where $\tau_{y z}^{i, i+1}$ is the shear stress at the interface between the $i^{\text {th }}$ and $i+1^{\text {th }}$ plies, and $z^{i-1, i}$ is the value of the $z$ coordinate at the interface between the $i-1^{\text {th }}$ and $i^{\text {th }}$ laminae. Crucial to the analysis is finding averaged displacements in the laminate for a given in-plane loading condition. An average of the mechanical parameters across the lamina is defined as

$$
\bar{\phi}^{(i)}=\frac{1}{h^{(i)}} \int_{h^{(i)}} \phi^{(i)} d z
$$

where an over-line denotes an averaged quantity. Notice that the average shown in Eq. (6) is for the ply $(i)$ and should not be confused with the average for the entire laminate which will be denoted by $\overline{\bar{\phi}}$. The overall reduced stiffness properties can be obtained applying unit normal and shear loads and calculating the induced deformations. The analysis begins by stating the fundamental equations (Hooke's law and equilibrium equations) in terms of averaged quantities. The Hooke's law for in-plane stresses can be written in terms of averaged in-plane displacements, after applying condition (4), as

$$
\begin{aligned}
& \bar{\sigma}_{x}^{(i)}=Q_{11}^{(i)} \bar{u}_{, x}^{(i)}+Q_{12}^{(i)} \bar{v}_{, y}^{(i)}+Q_{16}^{(i)}\left(\bar{u}_{, y}^{(i)}+\bar{v}_{, x}^{(i)}\right) \\
& \bar{\sigma}_{y}^{(i)}=Q_{21}^{(i)} \bar{u}_{, x}^{(i)}+Q_{22}^{(i)} \bar{v}_{, y}^{(i)}+Q_{26}^{(i)}\left(\bar{u}_{, y}^{(i)}+\bar{v}_{, x}^{(i)}\right) \\
& \bar{\tau}_{x y}^{(i)}=Q_{61}^{(i)} \bar{u}_{, x}^{(i)}+Q_{62}^{(i)} \bar{v}_{, y}^{(i)}+Q_{66}^{(i)}\left(\bar{u}_{, y}^{(i)}+\bar{v}_{, x}^{(i)}\right)
\end{aligned}
$$

where ( ), ${ }_{x}$ and ( ), represent partial derivatives with respect to $x$ and $y$ directions, respectively, and $Q_{i j}^{(i)}$ are the stiffness matrix of a lamina in the oblique coordinate system. The transformation of the properties from orthogonal to oblique coordinate system is described in Appendix A. Hooke's law for out-of-plane shear strains and stresses can be expressed in terms of interface shear stresses and averaged displacements by taking a weighted average of these equations (see Appendix B). These equations are usually called 'shear lag' equations, and they are written as follows.

$$
\begin{aligned}
& \left\{\begin{array}{l}
\bar{u}^{(i)}-\bar{u}^{(i-1)} \\
\bar{v}^{(i)}-\bar{v}^{(i-1)}
\end{array}\right\}=\frac{h^{(i-1)}}{6}\left[\begin{array}{ll}
S_{45} & S_{55} \\
S_{44} & S_{45}
\end{array}\right]^{(i-1)}\left\{\begin{array}{l}
\tau_{y z}^{i-2, i-1} \\
\tau_{x z}^{i-2, i-1}
\end{array}\right\}+\left(\frac{h^{(i-1)}}{3}\left[\begin{array}{ll}
S_{45} & S_{55} \\
S_{44} & S_{45}
\end{array}\right]^{(i-1)}+\frac{h^{(i)}}{3}\left[\begin{array}{ll}
S_{45} & S_{55} \\
S_{44} & S_{45}
\end{array}\right]^{(i)}\right)\left\{\begin{array}{l}
\tau_{y z}^{i-1, i} \\
\tau_{x z}^{i-1, i}
\end{array}\right\} \\
& +\frac{h^{(i)}}{6}\left[\begin{array}{ll}
S_{45} & S_{55} \\
S_{44} & S_{45}
\end{array}\right]^{(i)}\left\{\begin{array}{l}
\tau_{y z}^{i, i+1} \\
\tau_{x z}^{i, i+1}
\end{array}\right\}
\end{aligned}
$$


Annals of Solid and Structural Mechanics, 1:29—40, 2010.

where $S_{44}, S_{45}$ and $S_{55}$ are the components of the out-of-plane shear compliance matrix in oblique coordinates (Appendix A). Finally, the equilibrium equations in the $x$ and $y$ direction can be written as

$$
\begin{aligned}
& \bar{\sigma}_{x, x}^{(i)}+\bar{\tau}_{x y, y}^{(i)}+\left(\tau_{x z}^{i, i+1}-\tau_{x z}^{i-1, i}\right) / h^{(i)}=0 \\
& \bar{\tau}_{x y, x}^{(i)}+\bar{\sigma}_{y, y}^{(i)}+\left(\tau_{y z}^{i, i+1}-\tau_{y z}^{i-1, i}\right) / h^{(i)}=0
\end{aligned}
$$

Replacing equations (7) and (8) into equations (9) leads to a second-order system of partial differential equations in terms of the ply-averaged displacements. The analytical solution of that system can be expressed as

$$
\left\{\begin{array}{c}
\bar{u}^{(1)} \\
\bar{u}^{(2)} \\
\vdots \\
\bar{u}^{(n)} \\
\bar{v}^{(1)} \\
\bar{v}^{(2)} \\
\vdots \\
\bar{v}^{(n)}
\end{array}\right\}=\left[\begin{array}{c}
a_{1} \\
a_{2} \\
\vdots \\
a_{n} \\
a_{n+1} \\
a_{n+2} \\
\vdots \\
a_{2 n}
\end{array}\right] \sinh (\beta x)+\left[\begin{array}{c}
b_{1} \\
b_{2} \\
\vdots \\
b_{n} \\
b_{n+1} \\
b_{n+2} \\
\vdots \\
b_{2 n}
\end{array}\right] \sinh (\kappa y)+\left[\begin{array}{c}
\varepsilon_{x}^{c} \\
\varepsilon_{x}^{c} \\
\vdots \\
\varepsilon_{x}^{c} \\
1 / 2 \gamma_{x y}^{c} \\
1 / 2 \gamma_{x y}^{c} \\
\vdots \\
1 / 2 \gamma_{x y}^{c}
\end{array}\right] x+\left[\begin{array}{c}
1 / 2 \gamma_{x y}^{c} \\
1 / 2 \gamma_{x y}^{c} \\
\vdots \\
1 / 2 \gamma_{x y}^{c} \\
\varepsilon_{y}^{c} \\
\varepsilon_{y}^{c} \\
\vdots \\
\varepsilon_{y}^{c}
\end{array}\right] y
$$

where $n$ is half the number of plies in the laminate; $\varepsilon_{x}^{c}, \varepsilon_{y}^{c}, \gamma_{x y}^{c}$ are constant deformations that appear during the integration procedure; and $a_{i}, b_{i}, \beta$ and $\kappa$ are unknown constants with lack of physical meaning. Replacing (10) into (9) leads to two eigenvalue systems where $a_{i}-\beta$ and $b_{i}-\kappa$ are the pairs of eigenvectors and eigenvalues for each system. The final solution is the linear combination of all particular solutions, as follows

$$
\left\{\begin{array}{c}
\bar{u}^{(1)} \\
\bar{u}^{(2)} \\
\vdots \\
\bar{u}^{(n)} \\
\bar{v}^{(1)} \\
\bar{v}^{(2)} \\
\vdots \\
\bar{v}^{(n)}
\end{array}\right\}=\sum_{j=1}^{2 n} A_{j}\left[\begin{array}{c}
a_{1} \\
a_{2} \\
\vdots \\
a_{n} \\
a_{n+1} \\
a_{n+2} \\
\vdots \\
a_{2 n}
\end{array}\right]_{j}\left[\begin{array}{c}
b_{1} \\
b_{2} \\
\vdots \\
b_{n} \\
b_{n+1} \\
b_{n+2} \\
\vdots \\
b_{2 n}
\end{array}\right]_{j} \sinh \left(\beta_{j} x\right)+\sum_{j=1}^{2 n} B_{j}\left[\begin{array}{c}
\varepsilon_{x}^{c} \\
\varepsilon_{x}^{c} \\
\vdots \\
\varepsilon_{x}^{c} \\
1 / 2 \gamma_{x y}^{c} \\
1 / 2 \gamma_{x y}^{c} \\
\vdots \\
1 / 2 \gamma_{x y}^{c}
\end{array}\right] x+\left[\begin{array}{c}
1 / 2 \gamma_{x y}^{c} \\
1 / 2 \gamma_{x y}^{c} \\
\vdots \\
1 / 2 \gamma_{x y}^{c} \\
\varepsilon_{y}^{c} \\
\varepsilon_{y}^{c} \\
\vdots \\
\varepsilon_{y}^{c}
\end{array}\right] y
$$

where $A_{j}, B_{j} \varepsilon_{x}^{c}, \varepsilon_{y}^{c}$ and $\gamma_{x y}^{c}$ are unknown quantities. However, two $\beta_{i}$ and two $\kappa_{i}$ eigenvalues are equal to zero, so the number of unknowns is reduced to $4 n-1$. To find the values of these constants, the following boundary conditions are used. First, stress-free conditions are assumed at the surfaces of the cracks

$$
\begin{aligned}
& \frac{1}{2 l_{1}} \int_{-l_{1}}^{l_{1}} \bar{\sigma}_{x}^{(\theta)} d y=0, \text { at } x= \pm l_{2} \\
& \frac{1}{2 l_{1}} \int_{-l_{1}}^{l_{1}} \bar{\tau}_{x y}^{(\theta)} d y=0, \text { at } x= \pm l_{2}
\end{aligned}
$$


Annals of Solid and Structural Mechanics, 1:29—40, 2010.

$$
\begin{aligned}
& \frac{1}{2 l_{2}} \int_{-l_{2}}^{l_{2}} \bar{\sigma}_{y}^{(\varphi)} d x=0, \text { at } y= \pm l_{1} \\
& \frac{1}{2 l_{2}} \int_{-l_{2}}^{l_{2}} \bar{\tau}_{x y}^{(\varphi)} d x=0, \text { at } y= \pm l_{1}
\end{aligned}
$$

where $\theta, \varphi$, are the labels for laminae with cracks along $y$ and $x$ directions, respectively (Figure 1). Equation (12) represents a set of $2 N_{\theta}+2 N_{\varphi}$ independent conditions, where $N_{\theta}$ and $N_{\varphi}$ are the number of $\theta$ - and $\varphi$-laminae, respectively. Second, the stresses in the uncracked surfaces are related to the external loads by

$$
\begin{gathered}
\sum_{u x=1}^{n-N_{\theta}} \frac{h^{(u x)}}{2 l_{1}} \int_{-l_{1}}^{l_{1}} \bar{\sigma}_{x}^{(u x)} d y=\overline{\bar{h}} \overline{\bar{\sigma}}_{x}, \text { at } x= \pm l_{2} \\
\sum_{u x=1}^{n-N_{\theta}} \frac{h^{(u x)}}{2 l_{1}} \int_{-l_{1}}^{l_{1}} \bar{\tau}_{x y}^{(u x)} d y=\overline{\bar{h}} \overline{\bar{\tau}}_{x y}, \text { at } x= \pm l_{2} \\
\sum_{u y=1}^{n-N_{\varphi}} \frac{h^{u y}}{2 l_{2}} \int_{-l_{2}}^{l_{2}} \bar{\sigma}_{y}^{(u y)} d x=\overline{\bar{h}} \overline{\bar{\sigma}}_{y}, \text { at } y= \pm l_{1}
\end{gathered}
$$

where $\overline{\bar{\sigma}}_{x}, \overline{\bar{\sigma}}_{y}$ and $\overline{\bar{\tau}}_{x y}$ are the components of the external load applied to the laminate; and $u x$ and $u y$ are the labels for uncracked ply surfaces normal to $x$ and $y$ directions, respectively. Three independent conditions are obtained from Eq. (13). Finally, the displacements in the $x$ and $y$ directions are assumed to be the same for all uncracked ply surfaces

$$
\begin{aligned}
& \frac{1}{l_{2}} \int_{0}^{l_{2}} \bar{u}^{(u y r)} d x=\frac{1}{l_{2}} \int_{0}^{l_{2}} \bar{u}^{(u y)} d x \text { at } y= \pm l_{1} \quad \forall u y \neq u y r \\
& \frac{1}{l_{2}} \int_{0}^{l_{2}} \bar{v}^{(u y r)} d x=\frac{1}{l_{2}} \int_{0}^{l_{2}} \bar{v}^{(u y)} d x \text { at } y= \pm l_{1} \quad \forall u y \neq u y r \\
& \frac{1}{l_{1}} \int_{0}^{l_{1}} \bar{u}^{(u x r)} d y=\frac{1}{l_{1}} \int_{0}^{l_{1}} \bar{u}^{(u x)} d y \quad \text { at } x= \pm l_{2} \quad \forall u x \neq u x r \\
& \frac{1}{l_{1}} \int_{0}^{l_{1}} \bar{v}^{(u x r)} d y=\frac{1}{l_{1}} \int_{0}^{l_{1}} \bar{v}^{(u x)} d y \quad \text { at } x= \pm l_{2} \quad \forall u x \neq u x r
\end{aligned}
$$

where $(u x r)$ and $(u y r)$ are two uncracked laminae taken as reference. From Eqs. (14a) and (14b), the number of independent conditions that can be obtained is $2\left(n-N_{\varphi}-1\right)$; on the other hand, $2\left(n-N_{\theta}-1\right)$ conditions are obtained from Eqs. (14c) and (14d). Equations (12)-(14) represent the set of $4 n-1$ boundary conditions, which allows us to calculate all the unknowns. Then, the average strains can be calculated as

$$
\begin{gathered}
\overline{\bar{\varepsilon}}_{x}=\frac{1}{4 l_{1} l_{2}} \int_{-l_{1}}^{l_{1}} \int_{-l_{2}}^{l_{2}} \bar{u}_{, x}^{(\varphi)} d x d y \\
\overline{\bar{\varepsilon}}_{y}=\frac{1}{4 l_{1} l_{2}} \int_{-l_{1}}^{l_{1}} \int_{-l_{2}}^{l_{2}} \bar{v}_{, y}^{(\theta)} d x d y \\
\overline{\bar{\gamma}}_{x y}=\frac{1}{4 l_{1} l_{2}} \int_{-l_{1}}^{l_{1}} \int_{-l_{2}}^{l_{2}}\left(\bar{v}_{, x}^{(\varphi)}+\bar{u}_{, y}^{(\theta)}\right) d x d y
\end{gathered}
$$


To obtain the compliance matrix $S$ of the cracked composite laminate, three unit-load cases are considered

$$
\left\{\begin{array}{l}
\overline{\bar{\sigma}}_{x} \\
\overline{\bar{\sigma}}_{y} \\
\overline{\bar{\tau}}_{x y}
\end{array}\right\}^{(a)}=\left[\begin{array}{l}
1 \\
0 \\
0
\end{array}\right] ;\left\{\begin{array}{l}
\overline{\bar{\sigma}}_{x} \\
\overline{\bar{\sigma}}_{y}^{(b)} \\
\overline{\bar{\tau}}_{x y}
\end{array}\right\}=\left[\begin{array}{l}
0 \\
1 \\
0
\end{array}\right] \text { and }\left\{\begin{array}{l}
\overline{\bar{\sigma}}_{x} \\
\overline{\bar{\sigma}}_{y} \\
\overline{\bar{\tau}}_{x y}
\end{array}\right\}^{(c)}=\left[\begin{array}{l}
0 \\
0 \\
1
\end{array}\right] ;
$$

and the deformations obtained for each case are the components of the increased compliance matrix $\tilde{S}$ in the oblique coordinate system

$$
\left.\tilde{\mathbf{S}}=\left[\begin{array}{c}
\left\{\overline{\bar{\varepsilon}}_{x}\right. \\
\overline{\bar{\varepsilon}}_{y} \\
\overline{\bar{\gamma}}_{x y}
\end{array}\right\}^{(a)} \quad\left\{\begin{array}{l}
\overline{\bar{\varepsilon}}_{x} \\
\overline{\bar{\varepsilon}}_{y} \\
\overline{\bar{\gamma}}_{x y}
\end{array}\right\}^{(b)} \quad\left\{\begin{array}{l}
\overline{\bar{\varepsilon}}_{x} \\
\overline{\bar{\varepsilon}}_{y} \\
\overline{\bar{\gamma}}_{x y}
\end{array}\right\}\right]
$$

The compliance matrix in the orthogonal coordinate system, $\mathbf{S}$, is obtained using the coordinate transformation

$$
\mathbf{S}=\mathbf{T}^{-1} \tilde{\mathbf{S}} \mathbf{T}^{-T}, \quad \mathbf{T}=\left[\begin{array}{ccc}
\cos ^{2} \theta & \sin ^{2} \theta & \cos \theta \sin \theta \\
\cos ^{2} \varphi & \sin ^{2} \varphi & \cos \varphi \sin \varphi \\
2 \cos \theta \sin \varphi & 2 \cos \varphi \sin \theta & \cos \theta \sin \varphi \\
& & +\cos \varphi \sin \theta
\end{array}\right]
$$

The overall elastic properties for the cracked laminate can be written in terms of the components of the matrix $\mathbf{S}$ as

$$
E_{X}=\frac{1}{S_{11}}, E_{Y}=\frac{1}{S_{22}}, v_{X Y}=-\frac{S_{12}}{S_{11}} \text { and } G_{X Y}=\frac{1}{S_{33}}
$$

A very important characteristic of the procedure presented here is that properties of the damaged lamina are function only of the original elastic properties and the crack densities $\lambda_{1}$ and $\lambda_{2}$. Therefore, there is not need for defining damage evolution functions in terms of additional, thus no need to adjust such parameters using additional experimental data.

\section{Fracture Evolution Analysis}

In the previous section, the elastic properties of the damaged laminate were calculated as a function of the crack densities. In this section the strain Energy Release Rate (ERR) and a damage evolution analysis are formulated based on the results presented above.

The ERR associated with Mode I and Mode II can be expressed as

$$
\begin{gathered}
G_{I}=-\frac{\partial U_{I}}{\partial A} \\
G_{I I}=-\frac{\partial U_{I I}}{\partial A}
\end{gathered}
$$

where $U_{I}$ and $U_{I I}$ are the strain energy for Mode I and Mode II, respectively; and $A$ is the crack area. The crack area of the $\varphi$ - and $\theta$-laminae depend on the crack densities $\lambda_{1}$ and $\lambda_{2}$, respectively. For $\theta$-cracks, $G_{I}$ and $G_{I I}$ can be expressed in terms of the crack density $\lambda_{2}$ as

$$
G_{I}=-\frac{\partial U_{I}^{\theta}}{\partial \lambda_{2}} \frac{\partial \lambda_{2}}{\partial A^{\theta}}
$$


Annals of Solid and Structural Mechanics, 1:29—40, 2010.

$$
G_{I I}=-\frac{\partial U_{I I}^{\theta}}{\partial \lambda_{2}} \frac{\partial \lambda_{2}}{\partial A^{\theta}} .
$$

where $A^{\theta}$ is the area of cracks in the $\theta$-laminae and $U_{I}^{\theta}$ and $U_{I I}^{\theta}$ are the Mode $\mathrm{I}$ and Mode II strain energies associated with the $\theta$-laminae. For $\varphi$-cracks, an expression similar to Eq. (21) can be easily obtained in terms of $\lambda_{1}$. For example, considering $\theta$ cracks, the strain energy components $U_{I}^{\theta}$ and $U_{I I}^{\theta}$ can be expressed in terms of the average (applied) stresses and strains in material coordinates as

$$
\begin{gathered}
G_{I}^{\theta}=-\frac{V_{u c}}{2} \frac{\partial\left\{\overline{\bar{\sigma}}_{1}^{\theta} \overline{\bar{\varepsilon}}_{1}^{\theta}+\overline{\bar{\sigma}}_{2}^{\theta} \overline{\bar{\varepsilon}}_{2}^{\theta}\right\}}{\partial \lambda_{2}} \frac{\partial \lambda_{2}}{\partial A^{\theta}} \\
G_{I I}^{\theta}=-\frac{V_{u c}}{2} \frac{\partial\left\{\overline{\bar{\tau}}_{12}^{\theta} \overline{\bar{\gamma}}_{12}^{\theta}\right\}}{\partial \lambda_{2}} \frac{\partial \lambda_{2}}{\partial A^{\theta}}
\end{gathered}
$$

where $V_{u c}$ is the volume of the unit cell. The stresses and strains in the material coordinates can be obtained by transformation from orthogonal coordinates

$$
\begin{gathered}
\left\{\begin{array}{c}
\overline{\bar{\sigma}}_{1}^{\theta} \\
\overline{\bar{\sigma}}_{2}^{\theta} \\
\overline{\bar{\tau}}_{12}^{\theta}
\end{array}\right\}=\left[\begin{array}{ccc}
\cos ^{2} \theta & \sin ^{2} \theta & 2 \cos \theta \sin \theta \\
\sin ^{2} \theta & \cos ^{2} \theta & -2 \cos \theta \sin \theta \\
-\cos \theta \sin \theta & \cos \theta \sin \theta & \cos ^{2} \theta-\sin ^{2} \theta
\end{array}\right]\left\{\begin{array}{c}
\overline{\bar{\sigma}}_{X} \\
\overline{\bar{\sigma}}_{Y} \\
\overline{\bar{\tau}}_{X Y}
\end{array}\right\}, \\
\left\{\begin{array}{c}
\overline{\bar{\varepsilon}}_{1}^{\theta} \\
\overline{\bar{\varepsilon}}_{2}^{\theta} \\
\overline{\bar{\gamma}}_{12}^{\theta} / 2
\end{array}\right\}=\left[\begin{array}{ccc}
\cos ^{2} \theta & \sin ^{2} \theta & 2 \cos \theta \sin \theta \\
\sin ^{2} \theta & \cos ^{2} \theta & -2 \cos \theta \sin \theta \\
-\cos \theta \sin \theta & \cos \theta \sin \theta & \cos ^{2} \theta-\sin ^{2} \theta
\end{array}\right]\left\{\begin{array}{c}
\overline{\bar{\varepsilon}}_{X} \\
\overline{\bar{\varepsilon}}_{Y} \\
\overline{\bar{\gamma}}_{X Y} / 2
\end{array}\right\},
\end{gathered}
$$

where $\overline{\bar{\sigma}}_{X}, \overline{\bar{\sigma}}_{Y}, \overline{\bar{\tau}}_{X Y}, \overline{\bar{\varepsilon}}_{X}, \overline{\bar{\varepsilon}}_{Y}$ and $\overline{\bar{\gamma}}_{X Y}$ are the components of the applied stresses and strains in the global (orthogonal) coordinates. In order to obtain a relation between the external loading and the crack density, a failure criterion must be applied. Several failure criteria in terms of ERR have been proposed for composite materials. In this study, the criterion proposed by Hahn (Hahn 1983) was chosen. It can be expressed as

$$
(1-r) \sqrt{\frac{G_{I}}{G_{I C}}}+r \frac{G_{I}}{G_{I C}}+\frac{G_{I I}}{G_{I I C}} \leq 1,
$$

where $r=G_{I C} / G_{I I C}$ and $G_{I C}$ and $G_{I I C}$ are the critical values of the ERR for Mode I and II.

The procedure shown in Eqs. (20)-(24) can be used to predict the crack evolution on symmetric laminates with otherwise arbitrary LSS under general loading. The procedure is illustrated first for the particular case of balanced laminates and later for unbalanced laminates. For balanced laminates the cracked plies will have opposite angles $(\varphi=-\theta)$. Additionally, if only axial loading is applied, the applied stress and the average strains reduce to

$$
\left\{\begin{array}{c}
\overline{\bar{\sigma}}_{X} \\
\overline{\bar{\sigma}}_{Y} \\
\overline{\bar{\tau}}_{X Y}
\end{array}\right\}=\left\{\begin{array}{c}
E_{X} \overline{\bar{\varepsilon}}_{X} \\
0 \\
0
\end{array}\right\},
$$


Annals of Solid and Structural Mechanics, 1:29—40, 2010.

$$
\left\{\begin{array}{c}
\overline{\bar{\varepsilon}}_{X} \\
\overline{\bar{\varepsilon}}_{Y} \\
\overline{\bar{\gamma}}_{X Y}
\end{array}\right\}=\left\{\begin{array}{c}
\overline{\bar{\varepsilon}}_{X} \\
-v_{X Y} \overline{\bar{\varepsilon}}_{X} \\
0
\end{array}\right\} .
$$

Notice that the stresses and strains in material coordinates (Eq. (23)) for the $\theta$ and $-\theta$ plies will have the same magnitude; therefore, it is expected that the $\theta$ and $-\theta$ plies develop the same crack density $\left(\lambda_{1}=\lambda_{2}=\lambda\right)$. The ERR for mode I and II can be obtained by substituting Eqs. (23) and (25) into Eq. (22)

$$
\begin{gathered}
G_{I}=\frac{V_{u c}}{2}\left[\frac{\partial E_{X}}{\partial \lambda}\left(\cos ^{4} \theta-2 v_{X Y} \sin ^{2} \theta \cos ^{2} \theta+\sin ^{4} \theta\right)-E_{X}\left(2 \frac{\partial v_{X Y}}{\partial \lambda} \sin ^{2} \theta \cos ^{2} \theta\right)\right] \frac{\partial \lambda}{\partial A} \overline{\bar{\varepsilon}}_{X}^{2} \\
G_{I I}=\frac{V_{u c}}{2}\left[\frac{\partial E_{X}}{\partial \lambda}\left(2\left(1+v_{X Y}\right)\right) \sin ^{2} \theta \cos ^{2} \theta+2 \frac{\partial v_{X Y}}{\partial \lambda} E_{X} \sin ^{2} \theta \cos ^{2} \theta\right] \frac{\partial \lambda}{\partial A} \overline{\bar{\varepsilon}}_{X}^{2}
\end{gathered}
$$

The normalized ERR for Modes I and II can be defined as

$$
\begin{gathered}
\bar{G}_{I}(\lambda, \theta)=\frac{G_{I}}{\overline{\bar{\varepsilon}}_{X}^{2}} \\
\bar{G}_{I I}(\lambda, \theta)=\frac{G_{I I}}{\overline{\bar{\varepsilon}}_{X}^{2}} .
\end{gathered}
$$

A fracture evolution equation can be obtained replacing Eq. (27) into Eq. (24) as follows

$$
(1-r) \sqrt{\frac{\bar{G}_{I}}{G_{I C}}} \overline{\bar{\varepsilon}}_{X}+\left(r \frac{\bar{G}_{I}}{G_{I C}}+\frac{\bar{G}_{I I}}{G_{I I C}}\right) \overline{\bar{\varepsilon}}_{X}^{2}=1
$$

Equation (28) represents the relation between the axial strain $\left(\overline{\bar{\varepsilon}}_{X}\right)$ and the crack density on the $\theta$-laminae. This equation is valid for symmetric balanced laminates under axial loading. Similar relations can be obtained for other failure criteria.

Another case of interest is the axial loading of unbalanced laminates $(\varphi \neq-\theta)$. In this case, the average (applied) stresses and strain can be expressed as

$$
\begin{gathered}
\left\{\begin{array}{c}
\overline{\bar{\sigma}}_{X} \\
\overline{\bar{\sigma}}_{Y} \\
\overline{\bar{\sigma}}_{X Y}
\end{array}\right\}=\left\{\begin{array}{c}
E_{X} \overline{\bar{\varepsilon}}_{X} \\
0 \\
0
\end{array}\right\}, \\
\left\{\begin{array}{c}
\overline{\bar{\varepsilon}}_{X} \\
\overline{\bar{\varepsilon}}_{Y} \\
\overline{\bar{\gamma}}_{X Y}
\end{array}\right\}=\left\{\begin{array}{c}
\overline{\bar{\varepsilon}}_{X} \\
-v_{X Y} \overline{\bar{\varepsilon}}_{X} \\
\eta_{X Y} \overline{\bar{\varepsilon}}_{X}
\end{array}\right\} .
\end{gathered}
$$

The ERR for modes I and II for the $\theta$-laminae is obtained using Eqs. (22), (23) and (29) as follows

$$
\begin{aligned}
& G_{I}^{\theta}=-\frac{V_{u c}}{2}\left[\frac{\partial E_{X}}{\partial \lambda_{2}}\left(\cos ^{4} \theta-2 \cos ^{2} \theta \sin ^{2} \theta v_{X Y}+\sin ^{4} \theta+\cos \theta \sin \theta\left(\cos ^{2} \theta-\sin ^{2} \theta\right) \eta_{X Y_{-} X}\right)\right. \\
& \left.+E_{X}\left(-2 \cos ^{2} \theta \sin ^{2} \theta \frac{\partial \nu_{X Y}}{\partial \lambda_{2}}+\cos \theta \sin \theta\left(\cos ^{2} \theta-\sin ^{2} \theta\right) \frac{\partial \eta_{X Y_{-} X}}{\partial \lambda_{2}}\right)\right] \frac{\partial \lambda_{2}}{\partial A} \overline{\bar{\varepsilon}}_{X}^{2}
\end{aligned}
$$


Annals of Solid and Structural Mechanics, 1:29—40, 2010.

$$
\begin{aligned}
& G_{I I}^{\theta}=-\frac{V_{u c}}{2}\left[\frac{\partial E_{X}}{\partial \lambda_{2}}\left(2 \cos ^{2} \theta \sin ^{2} \theta+2 \cos ^{2} \theta \sin ^{2} \theta v_{X Y}-\cos \theta \sin \theta\left(\cos ^{2} \theta-\sin ^{2} \theta\right) \eta_{X Y_{-} X}\right)\right. \\
& \left.+E_{X}\left(2 \cos ^{2} \theta \sin ^{2} \theta \frac{\partial v_{X Y}}{\partial \lambda_{2}}-\cos \theta \sin \theta\left(\cos ^{2} \theta-\sin ^{2} \theta\right) \frac{\partial \eta_{X Y_{-} X}}{\partial \lambda_{2}}\right)\right] \frac{\partial \lambda_{2}}{\partial A} \overline{\bar{\varepsilon}}_{X}^{2} .
\end{aligned}
$$

The crack evolution relation for the $\theta$-lamina is obtained substituting Eq. (30) into Eq. (24) as follows

$$
(1-r) \sqrt{\frac{\bar{G}_{I}^{\theta}}{G_{I C}}} \overline{\bar{\varepsilon}}_{X}+\left(r \frac{\bar{G}_{I}^{\theta}}{G_{I C}}+\frac{\bar{G}_{I I}^{\theta}}{G_{I I C}}\right) \overline{\bar{\varepsilon}}_{X}^{2}=1
$$

The same procedure can be followed in order to obtain $G_{I}^{\varphi}$ and $G_{I I}^{\varphi}$ and the crack evolution of the $\varphi$-laminae in terms of $\lambda_{1}$ as follows

$$
(1-r) \sqrt{\frac{\bar{G}_{I}^{\varphi}}{G_{I C}}} \overline{\bar{\varepsilon}}_{X}+\left(r \frac{\bar{G}_{I}^{\varphi}}{G_{I C}}+\frac{\bar{G}_{I I}^{\varphi}}{G_{I I C}}\right) \overline{\bar{\varepsilon}}_{X}^{2}=1
$$

Equations (31) and (32) describe the crack evolution for unbalanced laminates under unidirectional loading. Notice that Eqs. (31) and (32) must be satisfied simultaneously. Multiple $\mathrm{N}_{\theta}$ laminae with thickness $\mathrm{t}_{\theta}$ and angle $\theta$ as well as multiple $\mathrm{N}_{\varphi}$ laminae with thickness $\mathrm{t}_{\varphi}$ and angle $\varphi$ can be handled easily by applying $2 \mathrm{~N}_{\theta}+2 \mathrm{~N}_{\varphi}$ boundary conditions $\mathrm{Eq}(12)$ and taking into account that $\theta$-laminae and $\varphi$-laminae have crack densities $\lambda_{1}$ and $\lambda_{2}$, respectively.

\section{Results and Discussion}

In this section, comparisons between results obtained using the proposed formulation and experimental data reported in the literature are presented. The mechanical properties of the laminate as a function of crack density for several LSS are presented and compared with experiments. Finally, comparisons of damage evolution between analytical predictions and experimental results for several LSS are presented.

To validate the prediction of reduced mechanical properties due to the presence of obliquely-crossed matrix cracks, a comparison between analytical results from this formulation and that presented by Yokozeki and Aoki (2005) is presented in Fig. 2. For this comparison a $[ \pm 45]_{S}$ IM600/\#133 composite was selected. The material properties can be found in Table 1. The change of the Young's modulus and the Poisson's ratio was obtained as function of the crack density $\lambda_{2}$ of the $\theta=45^{\circ}$ lamina; the crack density $\lambda_{1}$ of the $\varphi=-45^{\circ}$ lamina was kept constant and equal to $1 \mathrm{crack} / \mathrm{mm}$ just to reproduce exactly the particular results presented by Yokozeki and Aoki (2005). A perfect match is observed between the two formulations. This was expected since the formulation presented here reduces to theirs for $\left[\theta_{\mathrm{m}} / \varphi_{\mathrm{n}}\right]_{\mathrm{S}}$ laminates.

Varna, et al. (1999) reported a series of experiments for unidirectional loading of $\left[0 / \pm \theta_{4} / 0_{1 / 2}\right]_{S}$ E-Glass/Epoxy laminates. The material properties for the plies can be found 
on Table 1. A comparison of normalized elastic modulus $E_{X}$ and Poisson's ratio $v_{X Y}$ for $\theta=90^{\circ}, 70^{\circ}$ and $55^{\circ}$ is presented in Figs. 3-5. The experimental data for $\theta=55^{\circ}$ reported by Varna et al. (1999) displays an offset from expected behavior. The authors of that study believed that shear damage was the cause of this offset. In this study, the data for the $55^{\circ}$ case (Fig. 5) was shifted by a constant value, so that the normalized values of $E_{X}$ and $v_{X Y}$ approach one when the crack density approaches zero. In general, good agreement was observed between the experiments and analytical results. The prediction of the properties for $\theta=70^{\circ}$ was acceptable, but not as good as those for $90^{\circ}$ and $55^{\circ}$. It is observed that the predicted values of $v_{X Y}$ for $\theta=55^{\circ}$ increase with crack density as expected. Yokozeki and Aoki (2005) also reported an increase of Poisson's ratio for $[ \pm \theta]_{\mathrm{S}}$ laminates for values of $\theta$ lower that $60^{\circ}$.

An important characteristic of the formulation presented here is that the prediction is function only of the crack density. Nuismer and Tan (1988) and Yokozeki and Aoki (2005) also presented formulations which are function only of the crack density. However, those formulations are limited $\left[ \pm \theta_{m}, 90_{n}\right]_{S}$ and $\left[\theta_{m} / \varphi_{n}\right]_{S}$ LSS, respectively. Thus, those are special cases of the formulation presented here for arbitrary LSS. Varna et al. (1999) and Singh and Talreja (2008) presented formulations for the prediction of the elastic properties of damaged laminates. Their methodology included finding several parameters using numerical and/or experimental results. In addition, their methods produced linear predictions for $\left[0 / \pm \theta_{4} / 0_{1 / 2}\right]_{S}$ composites, which may be inappropriate for large values of crack densities. It can be observed from the predictions of the formulation presented here that the values of $E_{X}$ and $v_{X Y}$ decrease asymptotically to a given value. This behavior is expected since the undamaging laminae eventually take the entire load. Linear predictions do not predict this expected behavior.

Comparison for the damage evolution (crack density vs. applied strain) between this formulation and experimental data reported by Varna et al. (1999) is presented in Figs. 68. The values of $G_{I C}$ and $G_{I I C}\left(175 \mathrm{~J} / \mathrm{m}^{2}\right.$ and $1500 \mathrm{~J} / \mathrm{m}^{2}$, respectively) were taken from the literature for experimental results for Glass Fiber/Epoxy composites (Rikards et al. 1998). A very good prediction of the damage initiation and evolution (crack density vs. strain) is obtained for all the cases. However, the prediction of the onset strain for the case of $70^{\circ}$ was not as good as those for the other angles. It is believed that this may be caused by numerical inaccuracies which were typically observed at small crack densities in laminates with large angles between the oblique coordinate axes.

The damage evolution for balanced laminates can be described by only one value of the crack density; which corresponds to the $90^{\circ}$ or $\pm \theta$ laminae. However, when the laminate is not balanced, each lamina may have its own cracking onset strain and crack density depending on the lamina angle and the applied load. An example of this behavior can be observed in Fig. 9, where an axial load was applied to a $\left[0 /-45_{4} / 55_{4} / 0_{1 / 2}\right]_{S}$ laminate with the materials properties of Varna et al. (1999) (Table 1). It can be observed that matrix cracks appear first in the $55^{\circ}$ lamina at a strain of $1.18 \%$. This was expected since the $55^{\circ}$ lamina has the largest tensile stress perpendicular to the fibers for axial loading. The 
Annals of Solid and Structural Mechanics, 1:29—40, 2010.

crack density of $55^{\circ}$ lamina increases until the $-45^{\circ}$ lamina starts cracking, at a strain of $1.64 \%$. After this point, the crack densities in the two laminae grow at different rates.

\section{Conclusion}

In this study, a model to predict material properties and damage evolution for symmetric laminates is presented. The formulation is valid for laminates with an arbitrary stacking sequence and matrix cracks in one or two directions. An important characteristic of this formulation is that changes on the elastic properties are predicted as a function of the crack densities only. Another important feature is that the evolution of damage is predicted using a strain energy release rate criterion without needing additional experimentally-adjusted parameters. Good comparison between the predictions and experiments is observed.

\section{Appendix A}

The covariant transformation of the strain tensor can be expressed in matrix form as

$$
\left[\begin{array}{ccc}
\bar{\varepsilon}_{x} & \bar{\varepsilon}_{x y} & \bar{\varepsilon}_{x z} \\
\bar{\varepsilon}_{x y} & \bar{\varepsilon}_{y} & \bar{\varepsilon}_{y z} \\
\bar{\varepsilon}_{x z} & \bar{\varepsilon}_{y z} & \bar{\varepsilon}_{z}
\end{array}\right]=\left[\begin{array}{ccc}
\cos \theta & \sin \theta & 0 \\
\cos \varphi & \sin \varphi & 0 \\
0 & 0 & 1
\end{array}\right]\left[\begin{array}{ccc}
\bar{\varepsilon}_{X} & \bar{\varepsilon}_{X Y} & \bar{\varepsilon}_{X Z} \\
\bar{\varepsilon}_{X Y} & \bar{\varepsilon}_{X} & \bar{\varepsilon}_{Y Z} \\
\bar{\varepsilon}_{X Z} & \bar{\varepsilon}_{Y Z} & \bar{\varepsilon}_{X}
\end{array}\right]\left[\begin{array}{ccc}
\cos \theta & \sin \theta & 0 \\
\cos \varphi & \sin \varphi & 0 \\
0 & 0 & 1
\end{array}\right]^{T}
$$

Equation (A1) can be rewritten using Voigt notation as

$$
\left\{\begin{array}{l}
\bar{\varepsilon}_{x} \\
\bar{\varepsilon}_{y} \\
\bar{\varepsilon}_{z} \\
\bar{\gamma}_{y z} \\
\bar{\gamma}_{x z} \\
\bar{\gamma}_{x y}
\end{array}\right\}=\left[\begin{array}{cccccc}
\cos ^{2} \theta & \sin ^{2} \theta & 0 & 0 & 0 & \cos \theta \sin \theta \\
\cos ^{2} \varphi & \sin ^{2} \varphi & 0 & 0 & 0 & \cos \varphi \sin \varphi \\
0 & 0 & 1 & 0 & 0 & 0 \\
0 & 0 & 0 & \sin \varphi & \cos \varphi & 0 \\
0 & 0 & 0 & \sin \theta & \cos \theta & 0 \\
2 \cos \theta \cos \varphi & 2 \sin \theta \sin \varphi & 0 & 0 & 0 & \sin \theta \cos \varphi
\end{array}\right]\left\{\begin{array}{l}
\bar{\varepsilon}_{X} \\
\bar{\varepsilon}_{Y} \\
\bar{\varepsilon}_{Z} \\
\bar{\gamma}_{Y Z} \\
\bar{\gamma}_{X Z} \\
\bar{\gamma}_{X Y}
\end{array}\right\}
$$

The contravariant transformation for the stresses can be expressed as

$$
\left\{\begin{array}{l}
\bar{\sigma}_{x} \\
\bar{\sigma}_{y} \\
\bar{\sigma}_{z} \\
\bar{\tau}_{y z} \\
\bar{\tau}_{x z} \\
\bar{\tau}_{x y}
\end{array}\right\}=\left[\begin{array}{cccccc}
\cos ^{2} \theta & \sin ^{2} \theta & 0 & 0 & 0 & \cos \theta \sin \theta \\
\cos ^{2} \varphi & \sin ^{2} \varphi & 0 & 0 & 0 & \cos \varphi \sin \varphi \\
0 & 0 & 1 & 0 & 0 & 0 \\
0 & 0 & 0 & \sin \varphi & \cos \varphi & 0 \\
0 & 0 & 0 & \sin \theta & \cos \theta & 0 \\
2 \cos \theta \cos \varphi & 2 \sin \theta \sin \varphi & 0 & 0 & 0 & \sin \theta \cos \varphi \\
\bar{c}_{X Z}
\end{array}\right]^{-T}\left\{\begin{array}{c}
\bar{\sigma}_{X} \\
\bar{\sigma}_{Y} \\
\bar{\sigma}_{Z} \\
\bar{\tau}_{Y Z} \\
\bar{\tau}_{X Z} \\
\bar{\tau}_{X Y}
\end{array}\right\} .
$$

Equations (A1)-(A3) are applicable to lamina-averaged, local or global stress and strain tensors. By means of Eqs. (A2) and (A3), the in-plane stiffness and compliance matrices for a lamina in the oblique coordinate system can be calculated, respectively, as follows

$$
\begin{gathered}
\mathbf{Q}=\mathbf{T}^{-T} \hat{\mathbf{Q}} \mathbf{T}^{-1}, \\
\mathbf{S}=\mathbf{T} \hat{\mathbf{S}} \mathbf{T}^{T},
\end{gathered}
$$


Annals of Solid and Structural Mechanics, 1:29—40, 2010.

where $\hat{\mathbf{Q}}$ and $\hat{\mathbf{S}}$ are the in-plane stiffness and compliance in the material (orthogonal) coordinate system of the undamaged lamina and

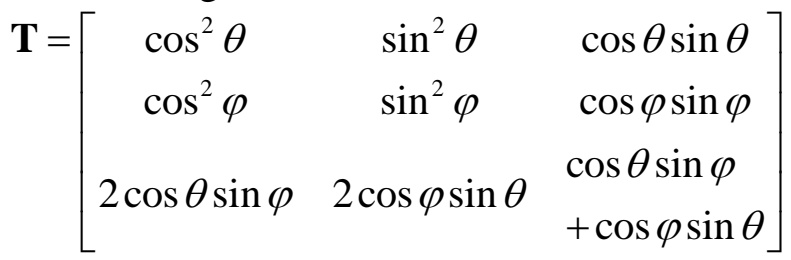

In a similar way, the out-of-plane shear stiffness and compliance matrices in the oblique coordinate system can be obtained

\section{Appendix B}

$$
\begin{gathered}
{\left[\begin{array}{ll}
Q_{44} & Q_{45} \\
Q_{45} & Q_{55}
\end{array}\right]=\left[\begin{array}{ll}
\cos \varphi & \sin \varphi \\
\cos \theta & \sin \theta
\end{array}\right]^{-T}\left[\begin{array}{ll}
\hat{Q}_{44} & \hat{Q}_{45} \\
\hat{Q}_{45} & \hat{Q}_{55}
\end{array}\right]\left[\begin{array}{cc}
\cos \varphi & \sin \varphi \\
\cos \theta & \sin \theta
\end{array}\right]^{-1}} \\
{\left[\begin{array}{ll}
S_{44} & S_{45} \\
S_{45} & S_{55}
\end{array}\right]=\left[\begin{array}{ll}
\cos \varphi & \sin \varphi \\
\cos \theta & \sin \theta
\end{array}\right]\left[\begin{array}{ll}
\hat{S}_{44} & \hat{S}_{45} \\
\hat{S}_{45} & \hat{S}_{55}
\end{array}\right]\left[\begin{array}{ll}
\cos \varphi & \sin \varphi \\
\cos \theta & \sin \theta
\end{array}\right]^{T} .}
\end{gathered}
$$

The relationship between the out-of-plane shear stresses and the averaged displacements can be obtained by calculating a weighted average of in-plane deformation. Using Hooke's law, the shear strains can be expressed in terms of the shear stresses as

$$
\begin{aligned}
& \gamma_{y z}^{(i)}=S_{44}^{(i)} \tau_{y z}^{(i)}+S_{45}^{(i)} \tau_{x z}^{(i)} . \\
& \gamma_{x z}^{(i)}=S_{45}^{(i)} \tau_{y z}^{(i)}+S_{55}^{(i)} \tau_{x z}^{(i)}
\end{aligned}
$$

Equation (B1) can be written in terms of displacements, and making use of Eqs. (3-4) the following equation is obtained

$$
\begin{aligned}
& u_{, z}^{(i)}+y_{, x}^{(j)^{0}}=S_{45}^{(i)}\left[\tau_{y z}^{i, i+1}+\left(\tau_{y z}^{i-1, i}-\tau_{y z}^{i, i+1}\right) \frac{h_{i}-z}{h^{(i)}}\right]+S_{55}^{(i)}\left[\tau_{x z}^{i, i+1}+\left(\tau_{x z}^{i-1, i}-\tau_{x z}^{i, i+1}\right) \frac{h_{i}-z}{h^{(i)}}\right] \\
& v_{, z}^{(i)}+y_{, y}^{\left(y^{0}\right.}=S_{44}^{(i)}\left[\tau_{y z}^{i, i+1}+\left(\tau_{y z}^{i-1, i}-\tau_{y z}^{i, i+1}\right) \frac{h_{i}-z}{h^{(i)}}\right]+S_{45}^{(i)}\left[\tau_{x z}^{i, i+1}+\left(\tau_{x z}^{i-1, i}-\tau_{x z}^{i, i+1}\right) \frac{h_{i}-z}{h^{(i)}}\right]
\end{aligned}
$$

The following weighted average is defined

$$
\frac{1}{h^{(i)}} \int_{h_{i-1}}^{h_{i}}()\left(h_{i}-z\right) d z
$$

Applying the operator shown in Eq. (B3) on both sides of Eq. (B2), the following relationship is obtained

$$
\begin{aligned}
& -u\left(h_{i-1}\right)+\bar{u}^{(i)}=\frac{h^{(i)}}{6} S_{45}^{(i)} \tau_{y z}^{i, i+1}+\frac{h^{(i)}}{3} S_{45}^{(i)} \tau_{y z}^{i-1, i}+\frac{h^{(i)}}{6} S_{55}^{(i)} \tau_{x z}^{i, i+1}+\frac{h^{(i)}}{3} S_{55}^{(i)} \tau_{x z}^{i-1, i} \\
& -v\left(h_{i-1}\right)+\bar{v}^{(i)}=\frac{h^{(i)}}{6} S_{44}^{(i)} \tau_{y z}^{i, i+1}+\frac{h^{(i)}}{3} S_{44}^{(i)} \tau_{y z}^{i-1, i}+\frac{h^{(i)}}{6} S_{45}^{(i)} \tau_{x z}^{i, i+1}+\frac{h^{(i)}}{3} S_{45}^{(i)} \tau_{x z}^{i-1, i}
\end{aligned}
$$

where $u\left(h_{i-1}\right)$ and $v\left(h_{i-1}\right)$ are the in-plane displacements evaluated at $z=h_{i-1}$. Following a similar procedure for ply $(i-1)$, but using $\left(z-h_{i-2}\right)$ as a weight, the following relation is obtained 
Annals of Solid and Structural Mechanics, 1:29—40, 2010.

$$
\begin{aligned}
& u\left(h_{i-1}\right)+\bar{u}^{(i-1)}=\frac{h^{(i-1)}}{6} S_{45}^{(i-1)} \tau_{y z}^{i-2, i-2}+\frac{h^{(i-1)}}{3} S_{45}^{(i-1)} \tau_{y z}^{i-1, i}+\frac{h^{(i-1)}}{6} S_{55}^{(i-1)} \tau_{x z}^{i-2, i-1}+\frac{h^{(i-1)}}{3} S_{55}^{(i-1)} \tau_{x z}^{i-1, i} \\
& v\left(h_{i-1}\right)+\bar{v}^{(i-1)}=\frac{h^{(i-1)}}{6} S_{44}^{(i-1)} \tau_{y z}^{i-2, i-1}+\frac{h^{(i-1)}}{3} S_{44}^{(i-1)} \tau_{y z}^{i-1, i}+\frac{h^{(i-1)}}{6} S_{45}^{(i-1)} \tau_{x z}^{i-2, i-1}+\frac{h^{(i-1)}}{3} S_{45}^{(i-1)} \tau_{x z}^{i-1, i}
\end{aligned}
$$

Adding Eqs (B4) and (B5) and presenting the result in a matrix form we obtain

$$
\begin{aligned}
& \left\{\begin{array}{l}
\bar{u}^{(i)}-\bar{u}^{(i-1)} \\
\bar{v}^{(i)}-\bar{v}^{(i-1)}
\end{array}\right\}=\frac{h^{(i-1)}}{6}\left[\begin{array}{ll}
S_{45} & S_{55} \\
S_{44} & S_{45}
\end{array}\right]^{(i-1)}\left\{\begin{array}{l}
\tau_{y z}^{i-2, i-1} \\
\tau_{x z}^{i-2, i-1}
\end{array}\right\}+\left(\frac{h^{(i-1)}}{3}\left[\begin{array}{cc}
S_{45} & S_{55} \\
S_{44} & S_{45}
\end{array}\right]^{(i-1)}+\frac{h^{(i)}}{3}\left[\begin{array}{cc}
S_{45} & S_{55} \\
S_{44} & S_{45}
\end{array}\right]^{(i)}\right)\left\{\begin{array}{c}
\tau_{y z}^{i-1, i} \\
\tau_{x z}^{i-1, i}
\end{array}\right\} \\
& \frac{h^{(i)}}{6}\left[\begin{array}{ll}
S_{45} & S_{55} \\
S_{44} & S_{45}
\end{array}\right]^{(i)}\left\{\begin{array}{c}
\tau_{y z}^{i, i+1} \\
\tau_{x z}^{i, i+1}
\end{array}\right\}
\end{aligned}
$$


Annals of Solid and Structural Mechanics, 1:29—40, 2010.

\section{Tables}

Table 1. Material properties for the laminates considered in study.

\begin{tabular}{lll}
\hline & $\begin{array}{l}\text { IM600/\#133 [Yokozeki } \\
\text { and Aoki 2005] }\end{array}$ & $\begin{array}{l}\text { E-Glass/Epoxy [Varna, } \\
\text { et al. 1999] }\end{array}$ \\
\hline$E_{\mathrm{L}}(\mathrm{GPa})$ & 147 & 44.7 \\
$E_{\mathrm{T}}(\mathrm{GPa})$ & 8.31 & 12.7 \\
$v_{\mathrm{LT}}$ & 0.352 & 0.297 \\
$v_{\mathrm{TT}}$ & $0.45^{*}$ & $0.41^{*}$ \\
$G_{\mathrm{LT}}(\mathrm{GPa})$ & 4.7 & 5.8 \\
Ply thickness $(\mathrm{mm})$ & 0.14 & 0.144 \\
\hline $\mathrm{G}_{\mathrm{IC}}\left(\mathrm{J} / \mathrm{m}^{2}\right)[$ Rikards et al. 1998] & 175 & 175 \\
$\mathrm{G}_{\mathrm{IIC}}\left(\mathrm{J} / \mathrm{m}^{2}\right)[$ Rikards et al. 1998] & 1500 & 1500 \\
\hline$*$ Assumed value. & &
\end{tabular}


Annals of Solid and Structural Mechanics, 1:29—40, 2010.

\section{Figures}
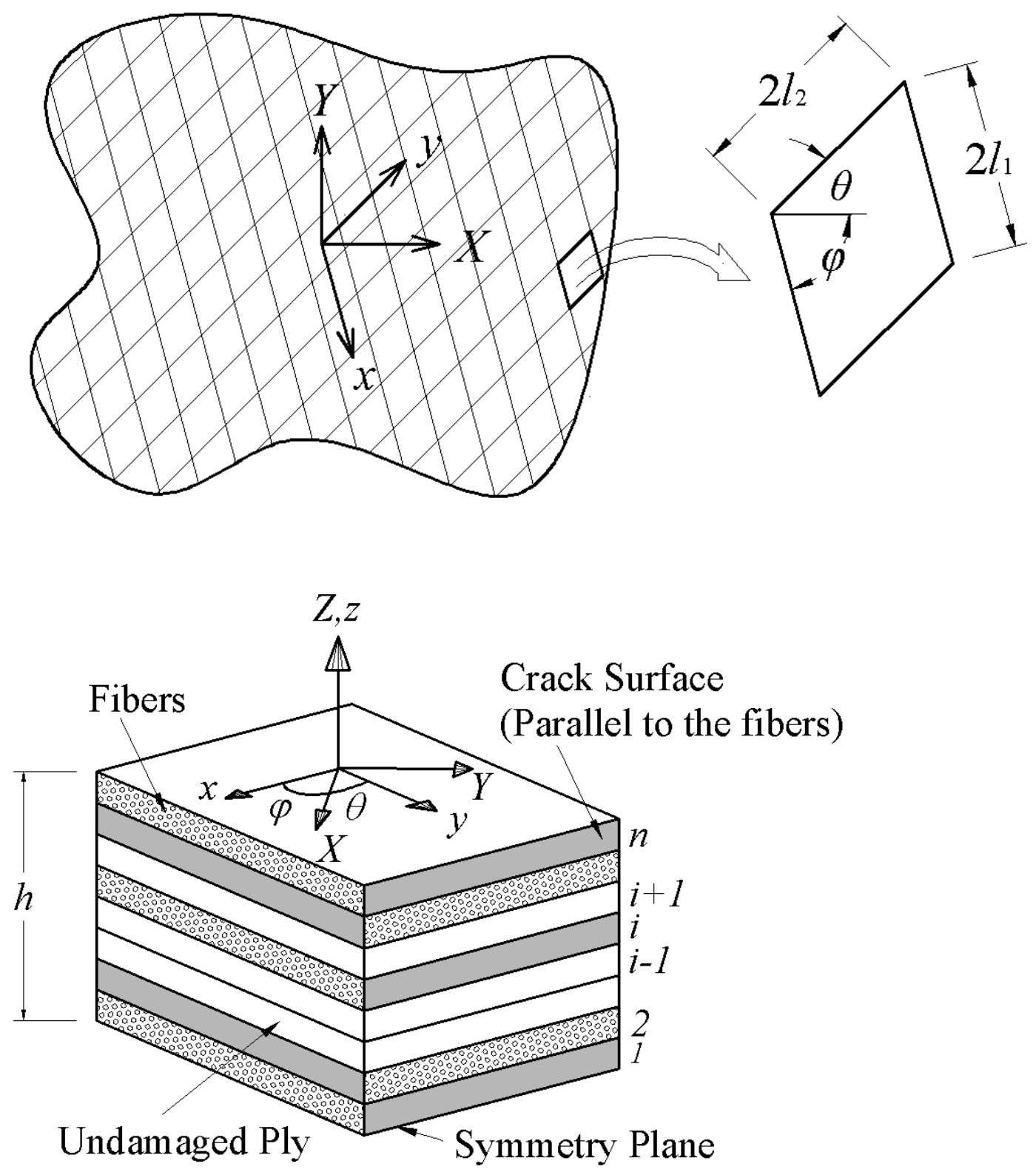

Figure 1. Orthogonal and oblique coordinate systems and geometry of the Repetitive Unit Cell. 
Annals of Solid and Structural Mechanics, 1:29—40, 2010.

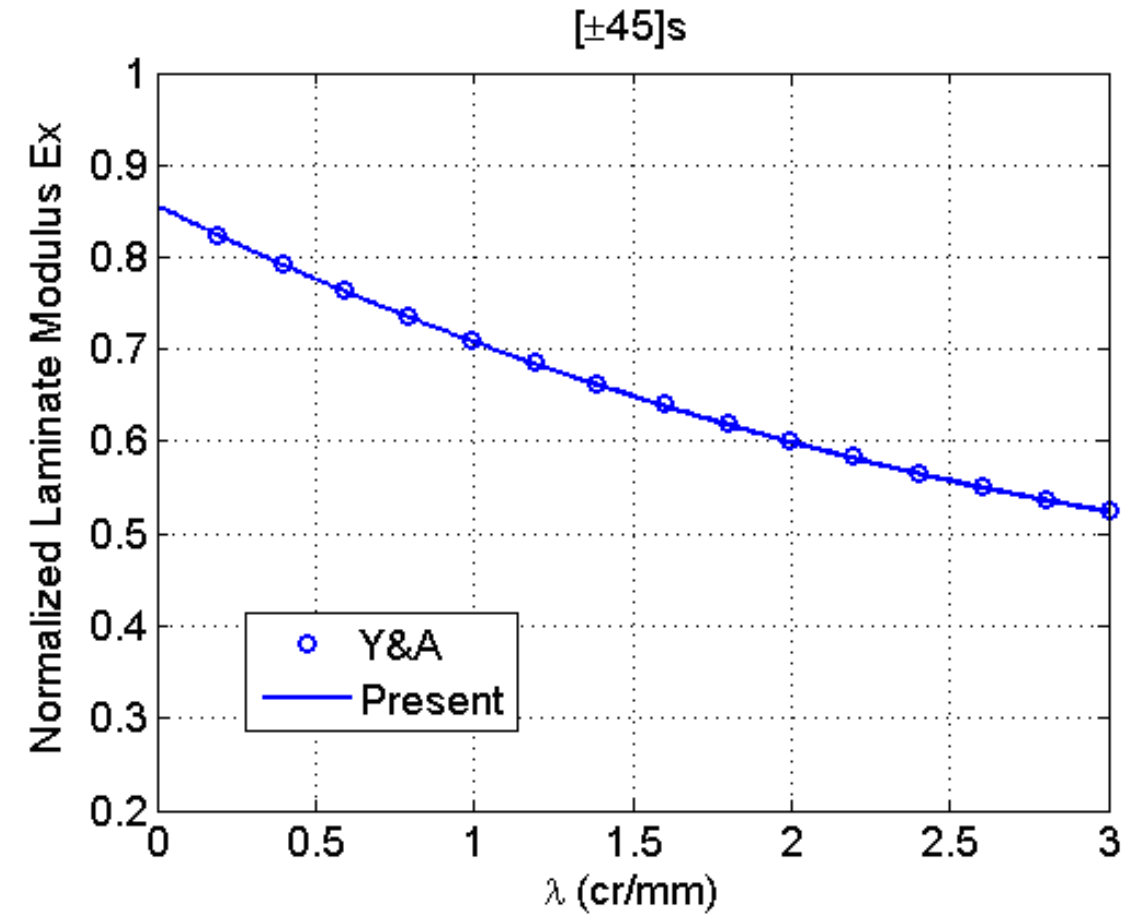

a)

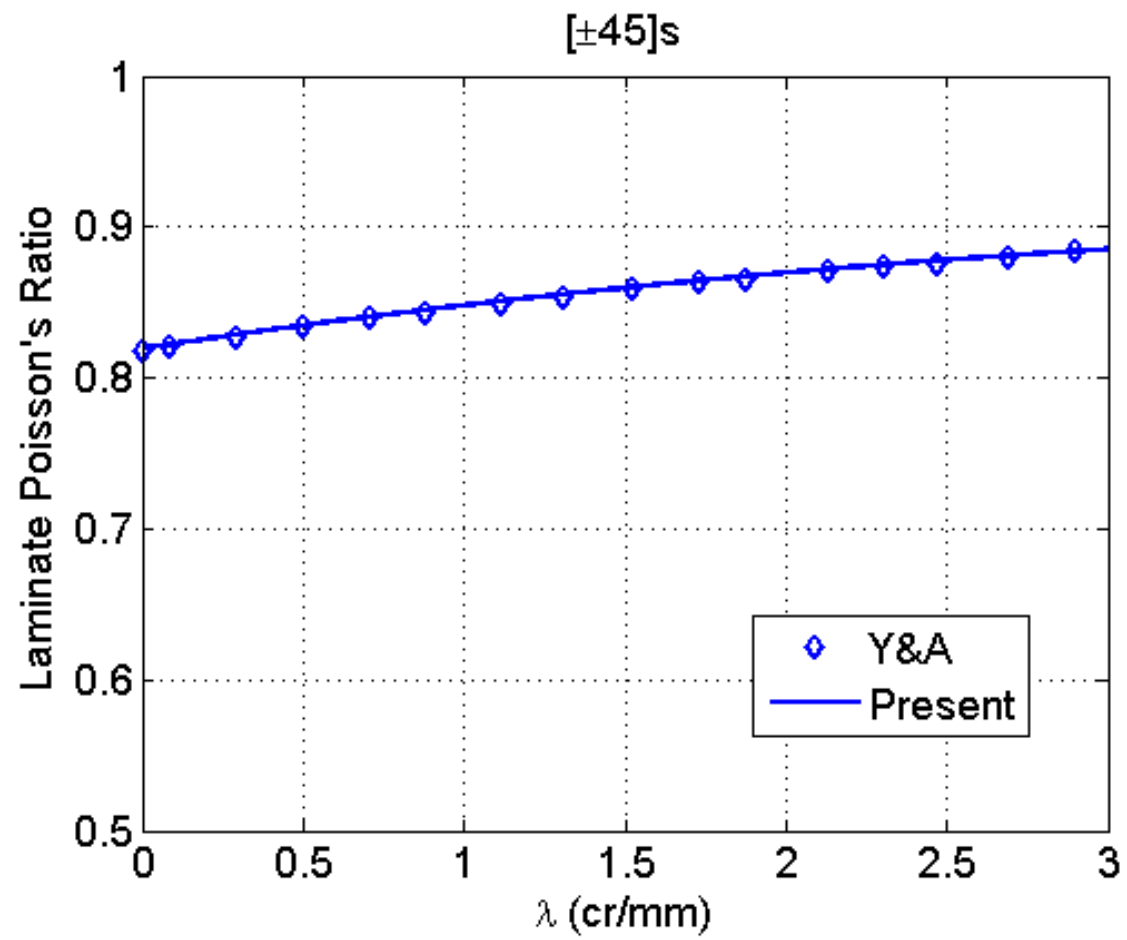

Figure 2. Comparison between analytic results from present formulation and those presented by (Yokozeki and Aoki 2005): a) Normalized Young's Modulus vs. $\lambda$, b) Laminate

Poisson's

Ratio

vs.

$\lambda$. 
Annals of Solid and Structural Mechanics, 1:29—40, 2010.

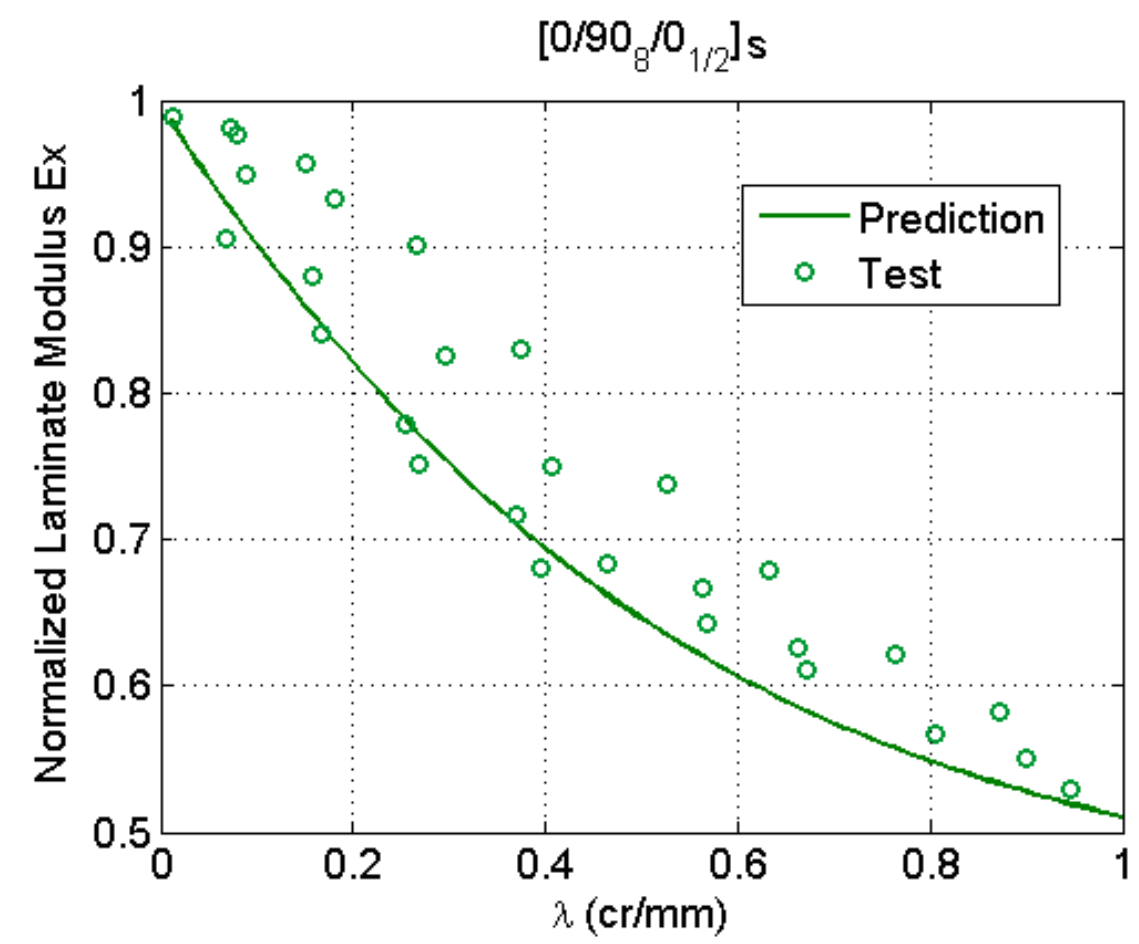

a)

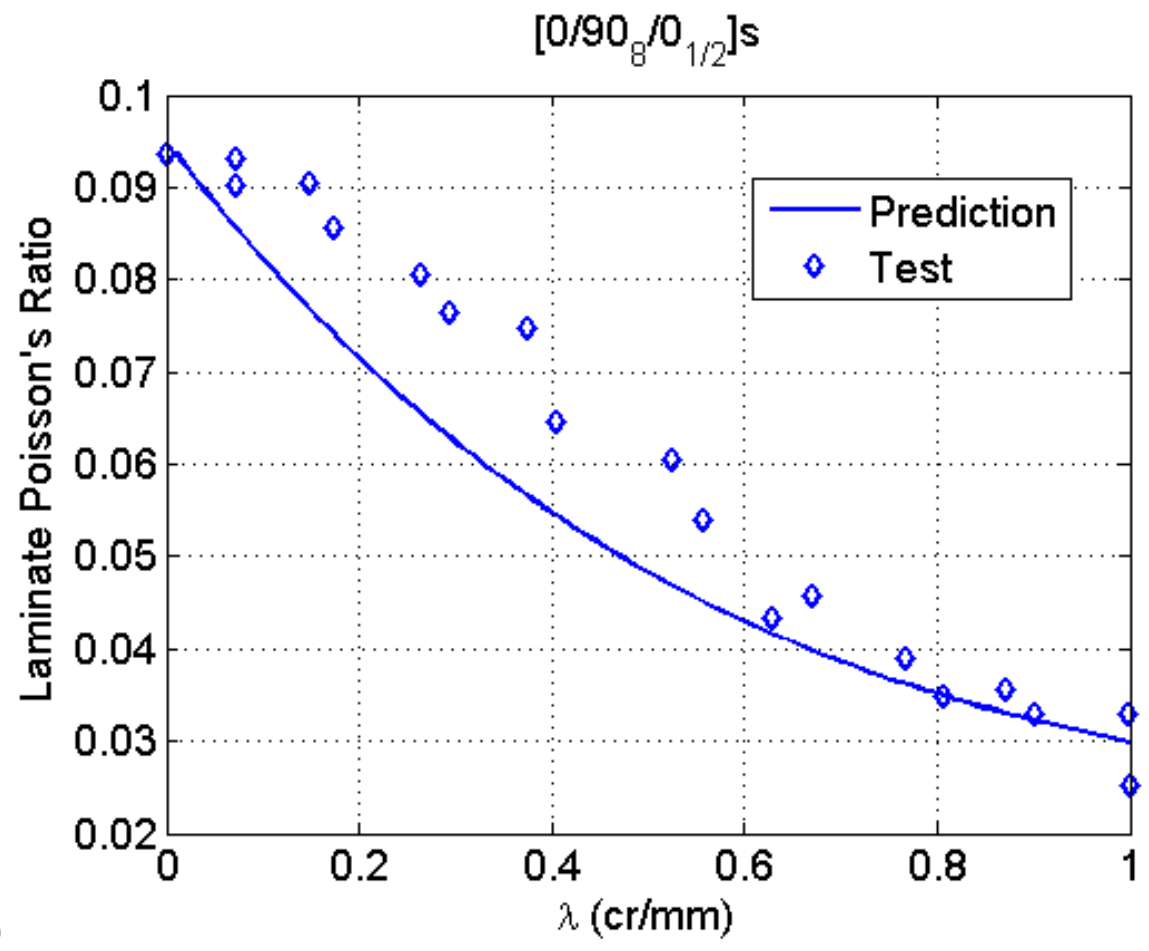

Figure 3. Reduced properties for the $\left[0 / 90_{8} / 0_{1 / 2}\right]$ s composite, a) Nomarlized Young's modulus $\left(\tilde{E}_{X} / E_{X}\right)$, b) Laminate Poisson's Ratio $\left(\tilde{v}_{X Y}\right)$. 
Annals of Solid and Structural Mechanics, 1:29—40, 2010.

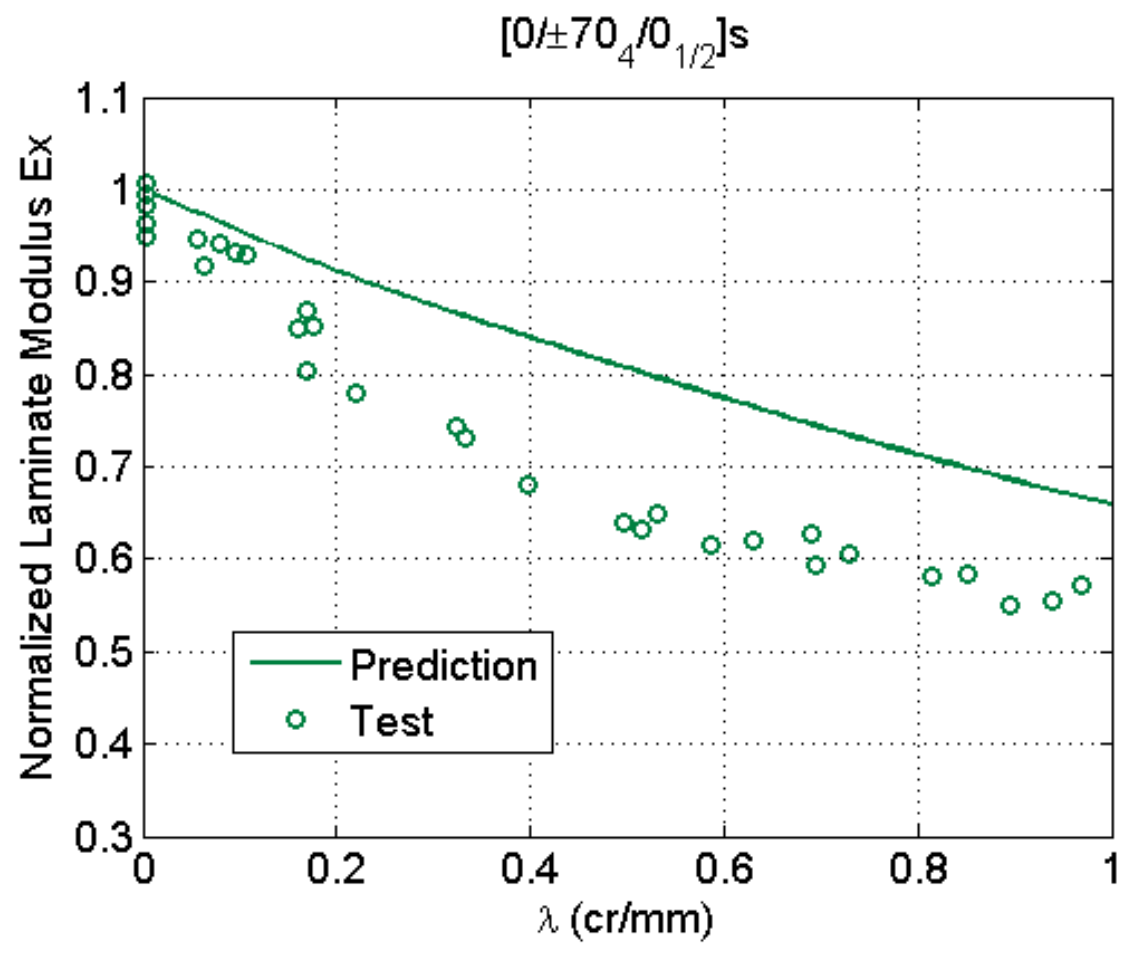

a)

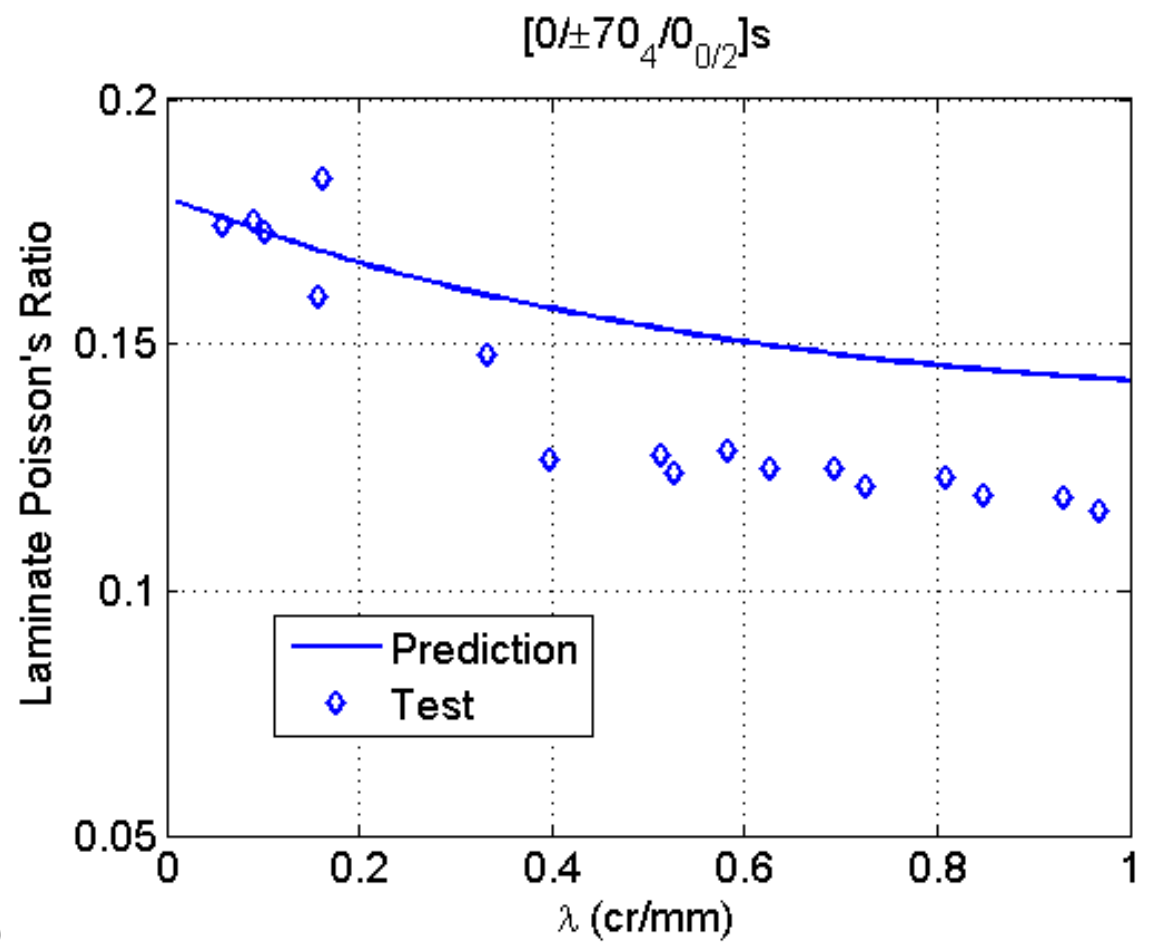

Figure 4. Reduced properties for the $\left[0 / \pm 70_{4} / 0_{1 / 2}\right] \mathrm{s}$ composite, a) Nomarlized Young's modulus $\left.\left(\tilde{E}_{X} / E_{X}\right), \mathrm{b}\right)$ Laminate Poisson's Ratio $\left(\tilde{v}_{X Y}\right)$. 
Annals of Solid and Structural Mechanics, 1:29—40, 2010.

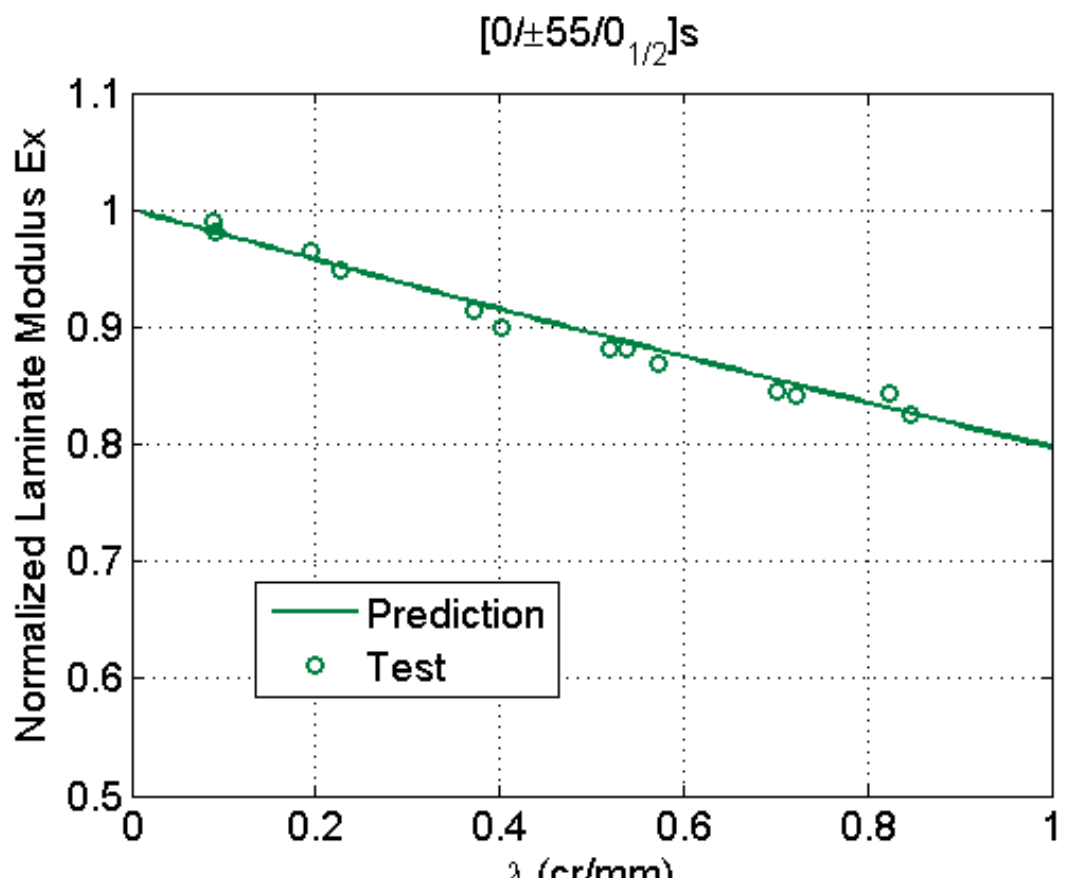

a)

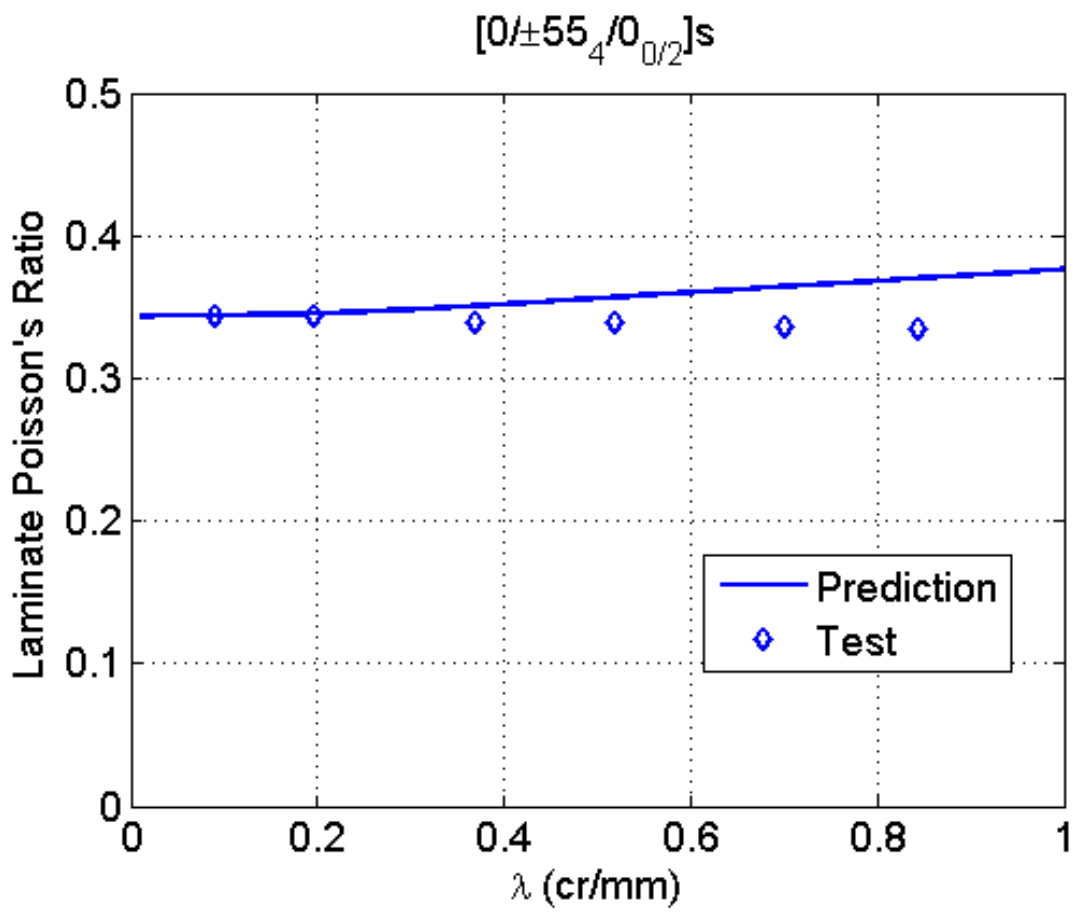

Figure 5. Reduced properties for the $\left[0 / \pm 55_{4} / 0_{1 / 2}\right]$ s composite, a) Nomarlized Young's modulus $\left(\tilde{E}_{X} / E_{X}\right)$, b) Laminate Poisson's Ratio $\left(\tilde{v}_{X Y}\right)$. 


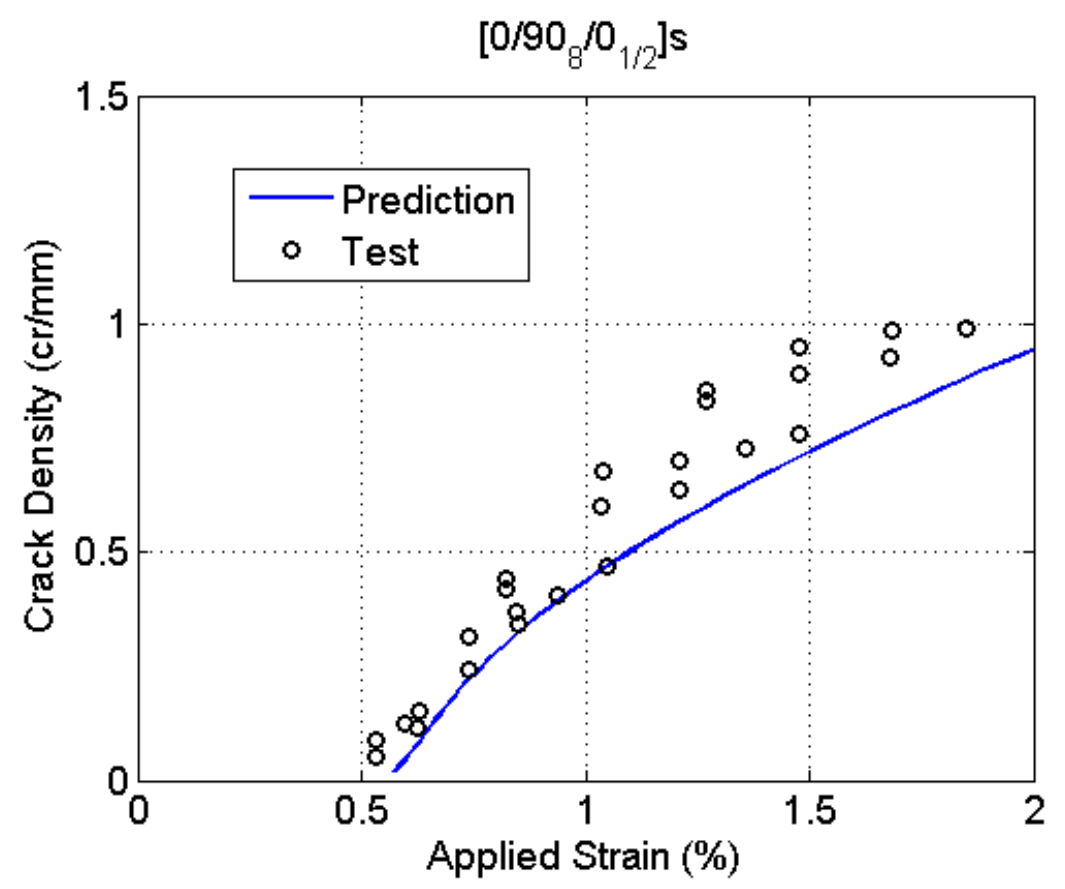

Figure 6. Comparison of the matrix crack evolution between analytical results and those reported by (Varna, et al. 1999). 


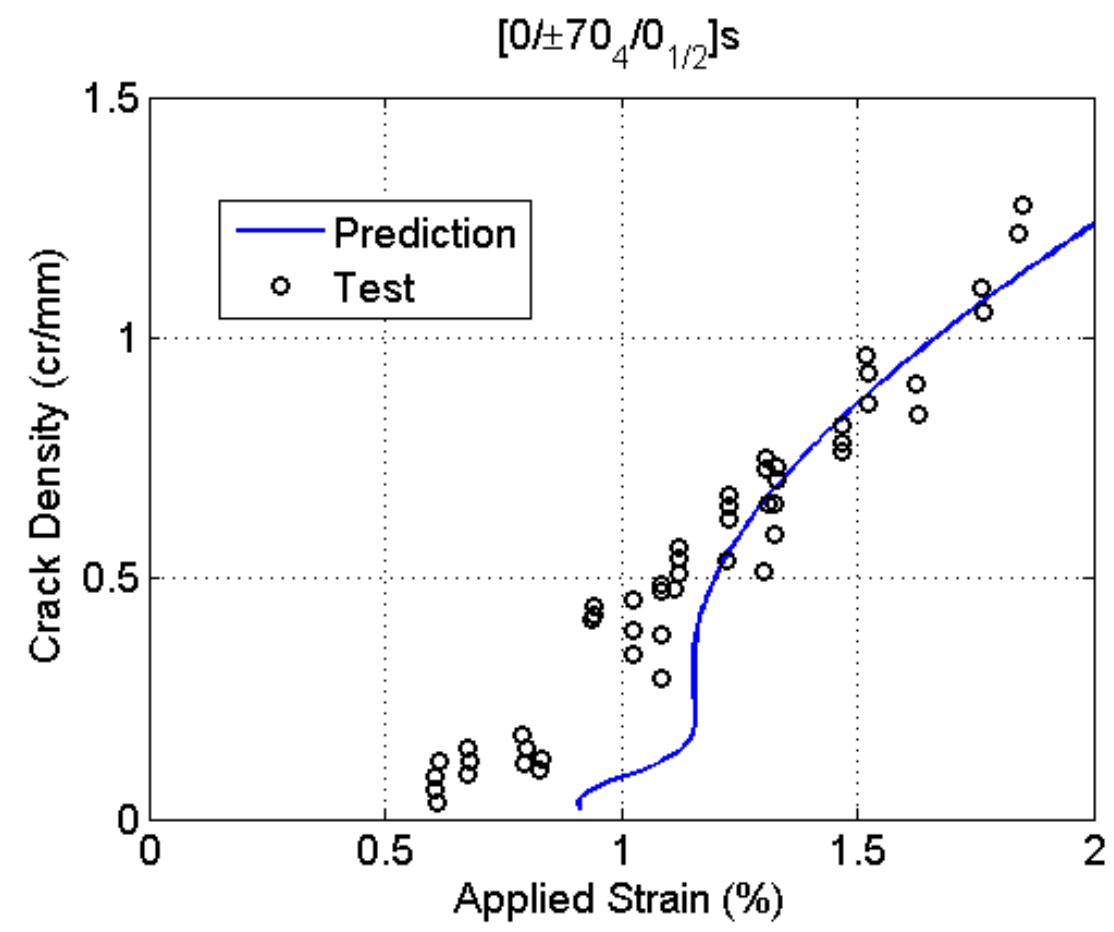

Figure 7. Comparison of the matrix crack evolution between analytical results and those reported by (Varna, et al. 1999).

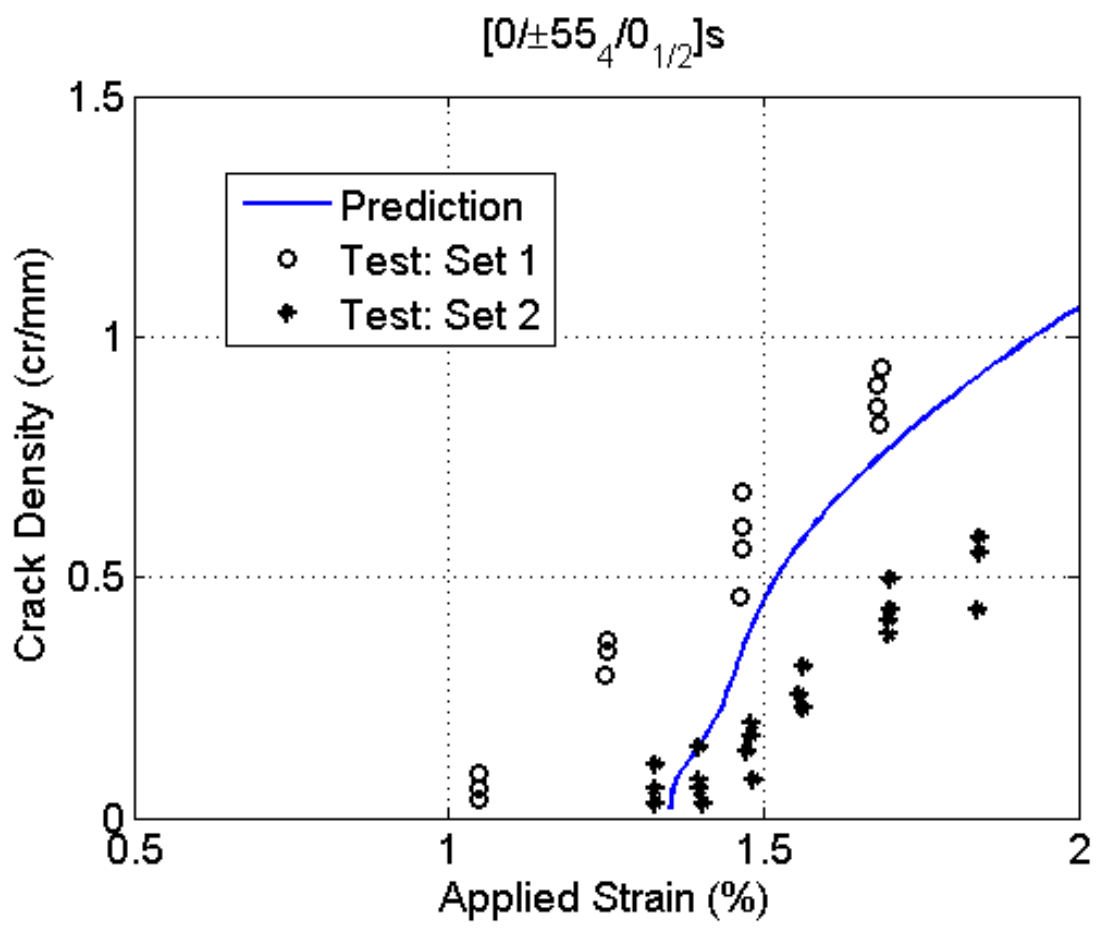

Figure 8. Comparison of the matrix crack evolution between analytical results and those reported by (Varna, et al. 1999). 
Annals of Solid and Structural Mechanics, 1:29—40, 2010.

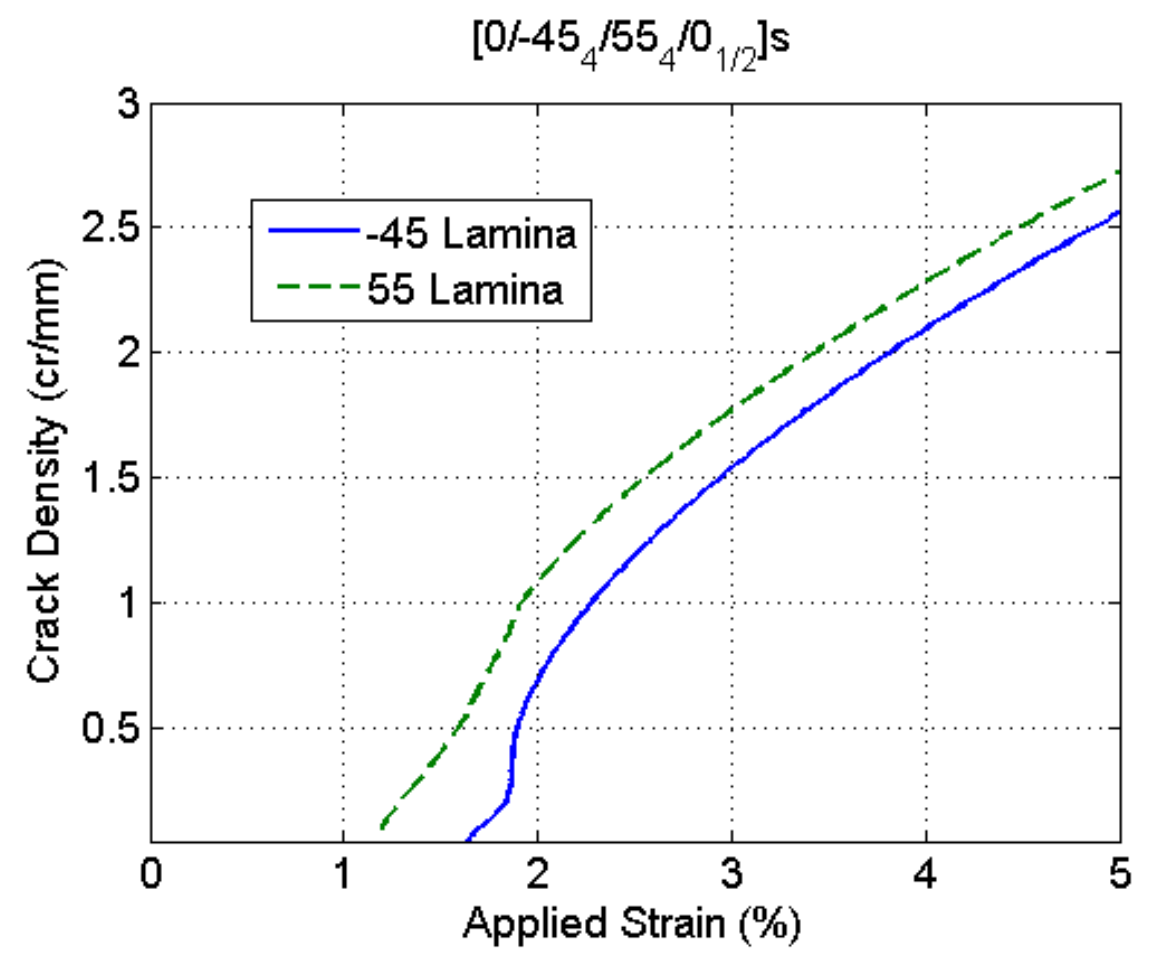

Figure 9. Damage evolution of a $\left[0 /-45_{4} / 55_{4} / 0_{1 / 2}\right]$ s laminate under axial loading. 
Annals of Solid and Structural Mechanics, 1:29—40, 2010.

\section{References}

Barbero E. J. (2007) Finite Element Analysis of Composite Materials. CRC Press, Boca Raton, FL

Barbero E. J., Abdelal G. F. and Caceres A. (2005) A micromechanics approach for damage modeling of polymer composites. Composite Structures 67:427-436

Barbero E. J. and DeVivo L. (2001) A constitutive model for elastic damage in fiberreinforced PMC laminae. Journal of Damage Mechanics 10:73-93

Barbero E. J. and Lonetti P. (2001) Damage model for composites defined in terms of available data. Mechanics of Composite Materials and Structures 8:299-316

Barbero E. J. and Lonetti P. (2002) An inelastic damage model for fiber reinforced laminates. Journal of Composite Materials 36:941-962

Camanho P. P., Davila C. G., Pinho S., et al. (2006) Prediction of in situ strengths and matrix cracking in composites under tension and in-plane shear. Composites Part A: Applied Science and Manufacturing 37:165-176

Davila C. G., Camanho P. P. and Rose C. A. (2005) Failure criteria for FRP laminates. Journal of Composite Materials 39:323-345

Fung Y. C. (1965) Foundations of Solid Mechanics. Prentice-Hall, Englewood Cliffs, NJ,USA

Hahn H. T. (1983) A mixed-mode fracture criterion for composite materials. Journal of Composites Technology and Research 5:26-29

Joffe R., krasnikovs A. and Varna J. (2001) COD-based simulation of transverse cracking and stiffness reduction in $\left[\mathrm{S} / 90_{\mathrm{n}}\right]_{\mathrm{s}}$ laminates. Composite Science and Technology 61:637-656

Kashtalyan M. and Soutis C. (2002) Analysis of local delaminations in composite laminates with angle-ply cracks. International Journal of Solids and Structures 39:1515-1537

Kashtalyan M. and Soutis C. (2007) Stiffness and fracture analysis of laminated composites with off-axis ply matrix cracking. Composites Part A: Applied Science and Manufacturing 38:1232-1269

Katerelos D. T. G., Varna J. and Galiotis C. (2007) Energy criterion for modelling damage evolution in cross-ply composite laminates. Composite Science and Technology In press

Li S., Reid S. R. and Soden P. D. (1998) A cotinuum damage model for transverse matrix cracking in laminate fibre-reinforced composites. Philosophical Transaction of the Royal Society of London. A 356:2379-2412

Lonetti P., Barbero E. J., Zinno R., et al. (2003) Interlaminar damage model for polymer matrix composites. Journal of Composite Materials 37:1485-1504

Lundmark P. and Varna J. (2005) Constitutive Relationships for Laminates with Ply Cracks in In-plane Loading. International Journal of Damage Mechanics 14:235259

Miami P., Camanho P. P., Mayugo J. A., et al. (2007a) A continuum damage model for composite laminates: Part I - Constitutive model. Mechanics of Materials 39:897908 
Annals of Solid and Structural Mechanics, 1:29—40, 2010.

Miami P., Camanho P. P., Mayugo J. A., et al. (2007b) A continuum damage model for composite laminates: Part II - Computation implementation and validation. Mechanics of Materials 39:909-919

Nairn J. (2000) Matrix Microcracking in Composites. In: R. Talreja and J. A. E. Manson (ed) Polymer Matrix Composites, Elservier Science, Amsterdan, pp 403-432

Nairn J. and Hu S. (2004) Matrix Microcracking. In: R. Talreja (ed) Damage Mechanics of Composites Materials, Elsevier, Amsterdan, pp 187-243

Nuismer R. J. and Tan S. C. (1988) Constitutive Relations of a Cracked Composite Lamina. Journal of Composite Materials 22:306-321

Rikards R., Buchholz F. G., Wang H., et al. (1998) Investigation of mixed mode I/II interlaminar fracture toughness of laminated composites by using a CTS type specimen. Engineering Fracture Mechanics 61:325-342

Schuecker C. and Pettermann H. E. (2006) A continuum damage model for fiber reinforced laminates based on ply failure mechanisms. Composites Structures 76:162-173

Singh C. V. and Talreja R. (2008) Analysis of Multiple Off-Axis Ply Cracks in Composite Laminates. International Journal of Solids and Structures 45:4574-4589

Talreja R. (1994) Damage Characterization by Internal Variables. In: R. Talreja (ed) Damage Mechanics of Composite Materials, Elsevier Science, Amsterdam, pp 5378

Tan S. C. and Nuismer R. J. (1989) A Theory for Progressive Matrix Cracking in Composite Laminates. Journal of Composite Materials 23:1029-1047

Varna J., Joffe R., Akshantala N. V., et al. (1999) Damage in Composite Laminates with Off-Axis Plies. Composite Science and Technology 59:2139-2147

Varna J., Joffe R. and Talreja R. (2001a) Mixed micromechanics and continuum damage mechanics approach to transverse cracking in $\left[\mathrm{S}, 90_{\mathrm{n}}\right]_{\mathrm{s}}$ laminates. Mechanics of Composite Materials 37:115-126

Varna J., Joffe R. and Talreja R. (2001b) A synergistic damage-mechanics analysis of transverse cracking in $\left[ \pm \theta / 90_{4}\right]_{\mathrm{s}}$ laminates. Composite Science and Technology 61:657-665

Yokozeki T. and Aoki T. (2005) Overall Thermoelastic Properties of Symmetric Laminates Containing Obliquely Crossed Matrix Cracks. Composite Science and Technology 65:1647-1654

Yokozeki T., Aoki T. and Ishikawa T. (2005a) Consecutive Matrix Cracking in Contiguous Plies of Composite Laminates. International Journal of Solids and Structures 42:2785-2802

Yokozeki T., Aoki T., Ogasawara T., et al. (2005b) Effects of layup angle and ply thickness on matrix crack interaction in contiguous plies of composite laminates. Composites Part A: Applied Science and Manufacturing 36:1229-1235

Zhang J., Fan J. and Soutis C. (1992) Analysis of multiple matrix cracking in $\left[ \pm \theta_{\mathrm{m}} / 90_{\mathrm{n}}\right]_{\mathrm{s}}$ composite laminates. Part 2: Development of transversely ply cracks. Composites 23:291-298 